\title{
1 Mapping the unique and shared functions of 2 oncogenic KRAS and RIT1 with proteome and 3 transcriptome profiling
}

4 April Lo ${ }^{1,2 *}$, Kristin Holmes ${ }^{1,2 *}$, Filip Mundt ${ }^{3,4,5}$, Sitapriya Moorthi ${ }^{1}$, Iris Fung ${ }^{3}$, Shaunt 5 Fereshetian $^{3}$, Jackie Watson ${ }^{3}$, Steven A. Carr ${ }^{3}$, Philipp Mertins ${ }^{3,6}$, Alice H. Berger ${ }^{1,2}$

1. Human Biology Division, Fred Hutchinson Cancer Research Center, Seattle, WA, USA

9 2. Genome Sciences Department, University of Washington, Seattle, WA, USA

10 3. Proteomics Platform, Broad Institute of MIT and Harvard, Cambridge, MA, USA

11 4. Department of Oncology-Pathology, Karolinska Institute, Stockholm, Sweden

12 5. Present address: Novo Nordisk Foundation center for Protein Research, Clinical

13 Proteomics, University of Copenhagen, Denmark

14 6. Present address: Proteomics Platform, Max Delbrück Center for Molecular Medicine

15 in the Helmholtz Society, Berlin, Germany 


\section{Abstract}

Aberrant activation of RAS oncogenes is prevalent in lung adenocarcinoma, with somatic mutation of KRAS occurring in $\sim 30 \%$ of tumors. Recently, we identified somatic mutation of the RAS-family GTPase RIT1 in lung adenocarcinoma, but relatively little is known about the

24 biological pathways regulated by RIT1 and how these relate to the oncogenic KRAS network.

25 Here we present quantitative proteomic and transcriptomic profiles from KRAS-mutant and RIT1-mutant isogenic lung epithelial cells and globally characterize the signaling networks regulated by each oncogene. We find that both mutant KRAS and mutant RIT1 promote S6 kinase, AKT, and RAF/MEK signaling, and promote epithelial-to-mesenchymal transition and immune evasion via HLA protein loss. However, KRAS and RIT1 diverge in regulation of phosphorylation sites on EGFR, USO1, and AHNAK proteins. The majority of the proteome changes are related to altered transcriptional regulation, but a small subset of proteins are differentially regulated by both oncoproteins at the post-transcriptional level, including

33 intermediate filament proteins, metallothioneins, and MHC Class I proteins. These data provide

34 the first global, unbiased characterization of oncogenic RIT1 network and identify the shared and divergent functions of oncogenic RIT1 and KRAS GTPases in lung cancer.

\section{Introduction}

38 Somatic mutation of the KRAS proto-oncogene is a prevalent feature of human cancers,

39 particularly in lung adenocarcinomas where KRAS is mutated in up to $30 \%$ of tumors. Cancer-

40 associated variants such as $\mathrm{G} 12 \mathrm{~V}$ and $\mathrm{Q} 61 \mathrm{H}$ alter the normal regulation of KRAS GTPase

41 activity by disrupting GTP hydrolysis activity or physical interaction with GTPase-activating

42 proteins (GAPs) $)^{1,2}$, resulting in heightened downstream cellular signaling through the canonical

43 RAS effector pathways RAF/MEK and PI3K/AKT as well as others. Following the discovery of 
44 cancer-associated RAS mutations in the $1980 \mathrm{~s}^{3,4}$, thousands of studies have delineated the

45 critical pathways involved in RAS-mediated cellular transformation, metastasis, and metabolism.

47 Recently, another RAS-family GTPase gene, RIT1, was found to harbor somatic mutations in

48 lung cancer ${ }^{5}$ and myeloid leukemias ${ }^{6}$. Interestingly, germline RIT1 mutations are found in

49 families with Noonan Syndrome, a developmental "RAS"-opathy involving altered craniofacial

50 morphology and cardiac abnormalities ${ }^{7}$, and which can also be caused by germline mutations in

51 KRAS itself or other RAS-pathway genes such as SOS1, SOS2, LZTR1 and SHOC2

52 (https://omim.org/). In cancer and development, RIT1 mutations are found in cases that lack

53 canonical KRAS mutations, suggesting that RIT1 may impart the same phenotypes conferred by

54 activation of RAS.

56 Prior studies have characterized the role of RIT1 in neural development ${ }^{8}$ and we and others

57 have described the role of mutant RIT1 in cellular transformation ${ }^{5,9,10}$, knowledge of the function

58 of cancer- and Noonan-associated RIT1 variants is relatively limited. Unlike KRAS, RIT1

59 mutations are rarely observed near the critical glycine residues involved in GTP hydrolysis (e.g.

60 G12 and G13 in KRAS or G30 and G31 in RIT1). Instead, RIT1 mutations occur most frequently

61 near the switch II domain, also targeted by Q61 KRAS variants (Fig. 1a). Nevertheless, these

62 mutations may enhance GTP-bound levels of RIT1 ${ }^{11,12}$. The molecular consequences of RIT1

63 switch II domain mutations may additionally be linked to the loss of RIT1's physical interaction

64 with LZTR1, a ubiquitin-conjugating enzyme responsible for degradation of RIT1 ${ }^{11}$. Cancer- and

65 Noonan-associated RIT1 variants lose the ability to interact with LZTR1 and consequently are

66 highly overexpressed, resulting in increased signaling activity through the RAF/MEK pathway ${ }^{11}$. 
68 Prior studies of RIT1 function focus on candidate cellular signaling pathways based on RIT1's

69 homology to KRAS. To our knowledge, unbiased mapping of downstream RIT1-regulated

70 pathways has not been performed to date. Here we sought to broadly describe the proteome,

71 phosphoproteome, and transcriptome changes induced by wild-type RIT1 and RIT1 ${ }^{\mathrm{M} 90 \mathrm{I}}$, a

72 cancer- and Noonan-associated variant, and to compare these changes to those induced by

73 oncogenic KRAS variants. With a particular interest in the consequences of RIT1 ${ }^{\mathrm{M} 901}$ in lung

74 cancer, we profiled the effects of RIT1 ${ }^{\mathrm{M} 901}$ mutation in AALE cells, a non-transformed,

75 immortalized, human lung epithelial cell line ${ }^{13}$.

77 By comparing the downstream pathways regulated by oncogenic KRAS and RIT1, we uncover

78 previously unknown consequences of RIT1 activation, such as induction of the epithelial-to-

79 mesenchymal transition (EMT) and post-translational regulation of HLA protein expression. In

80 addition, we uncover additional functional differences between KRAS and RIT1 including a

81 distinct and unique role of KRAS mutants in regulation of EGFR and USO1 phosphorylation.

82 These data provide the first systems-level view of RIT1 and RIT1 ${ }^{\mathrm{M} 90 \mathrm{l}}$ function.

\section{Results}

\section{Multi-omic profiling of RIT1- and RAS-transformed human lung epithelial cells}

87 We previously demonstrated that RIT1 ${ }^{\mathrm{M} 90 \mathrm{I}}$ and other cancer-associated RIT1 variants can

88 promote cellular transformation of NIH3T3 cells in vitro and in vivo ${ }^{5}$. To determine whether

89 RIT1 ${ }^{\mathrm{M} 901}$ was capable of transforming human lung epithelial cells, we expressed mutant RIT1 or

90 KRAS in the human lung epithelial cell line, AALE. Similar to our previous findings in rodent

91 cells, both RIT1 ${ }^{\mathrm{M} 90 \mathrm{I}}$ and KRAS ${ }^{\mathrm{G} 12 \mathrm{~V}}$ enabled AALE cells to form colonies in soft agar (Fig. 1b). 
93 The canonical function of oncogenic RAS variants is the downstream activation of the

94 RAF/MEK/ERK cellular signaling cascade ${ }^{14}$, and RIT1 shares the ability to bind C-RAF and

95 induce transcription of ERK target genes activity ${ }^{11}$. To determine if such regulation is active in

96 AALE cells, we stably expressed wild-type RIT1 or KRAS, or the mutant forms RIT1 ${ }^{\mathrm{M} 90 \mathrm{I}}$,

$97 \mathrm{KRAS}^{\mathrm{G} 12 \mathrm{~V}}$, and KRAS ${ }^{\mathrm{Q} 61 \mathrm{H}}$ in AALE cells. KRAS ${ }^{\mathrm{Q} 61 \mathrm{H}}$ was included since this mutant more closely

98 resembles the switch II domain mutants observed in RIT1 in cancer (Fig. 1a). RIT1 ${ }^{\text {M90I }}$,

$99 \mathrm{KRAS}^{\mathrm{G} 12 \mathrm{~V}}$, and $\mathrm{KRAS}^{\mathrm{Q} 61 \mathrm{H}}$ all enhanced ERK phosphorylation compared to their respective wild-

100 type protein or vector control (Fig. 1c). Interestingly, wild-type RIT1 overexpression also

101 modestly enhanced ERK phosphorylation whereas wild-type KRAS suppressed basal ERK

102 phosphorylation.

103

104 To systematically characterize the signaling networks perturbed by mutant RIT1 and KRAS in

105 lung cancer, we expressed each variant in AALE cells and performed both RNA-seq and deep

106 proteome and phosphoproteome profiling by liquid chromatography tandem mass spectrometry

107 (LC-MS/MS). Following trypsin digestion, peptides were labeled with tandem mass tag (TMT)

108 reagents in two overlapping 10-plex sets for relative quantification of proteome and

109 phosphopeptides by LC-MS/MS (Fig. 1d and Supplementary Fig. 1a-b). Following basic

110 reverse phase chromatography, fractions were either directly subjected to LC-MS/MS for total

111 proteome quantification, or subjected to immobilized metal affinity chromatography (IMAC) to

112 enrich for phosphopeptides and then subjected to LC-MS/MS, or. In total, we identified 10,131

113 proteins, 9002 of which were detected and quantified in every sample, and 29,140

114 phosphopeptides, 12,325 of which were identified in common in every sample (Supplementary

\section{Tables 1 and 2 and Supplementary Files 1 and 2).}


117 In parallel, we generated deep transcriptome profiles of the same isogenic cell lines.

118 Transcriptome profiling was performed in triplicate on the Illumina NovaSeq platform to a

119 median read-depth per replicate of 70.1 million reads (Fig. 1e, Supplementary Table 3 and

120 Supplementary Fig. 1e). No compensatory feedback regulation of RIT1 to KRAS or vice versa

121 was observed (Fig. 1f). Despite relatively low protein expression of KRAS variants in the AALE

122 lines (Fig. 1c), the majority of KRAS transcripts in each isogenic cell line corresponded to G12V

123 or $\mathrm{Q} 61 \mathrm{H}$ variants, respectively, with $84.1 \%$ of reads harboring the $\mathrm{G} 12 \mathrm{~V}$ variant in $\mathrm{KRAS}^{\mathrm{G} 12 \mathrm{~V}}$

124 cells, and $73.3 \%$ of reads corresponding to the $\mathrm{Q} 61 \mathrm{H}$ allele in KRAS ${ }^{\mathrm{Q} 61 \mathrm{H}}$ cells (Fig. 19). As

125 expected, known KRAS-regulated gene sets were strongly up- and down-regulated in KRAS-

126 mutant cells (Supplementary Fig. 1d).

Multi-omic profiling identifies global similarity between signaling regulated by RIT1 ${ }^{\text {M90I }}$

130 Differentially abundant proteins were identified by comparison to the vector control cells using a

131 two-tailed moderated t-test (Fig. 2a). Selected proteins observed to be significantly modulated

132 by LC-MS/MS were cross-validated by Western blot. FOSL1, also known as FRA1, is a basic

133 leucine zipper transcription factor in the FOS family ${ }^{15}$. Activation of RAS is known to promote

134 transcriptional upregulation and protein stabilization of FOSL $1^{16,17}$. By LC-MS/MS, FOSL1 was

135 markedly overexpressed in $\mathrm{KRAS}^{\mathrm{G} 12 \mathrm{~V}}, \mathrm{KRAS}^{\mathrm{Q} 61 \mathrm{H}}$, and RIT1 ${ }^{\mathrm{M} 90 \mathrm{I}}$-mutant cells compared to wild-

136 type cells or vector control cells (Fig. 2b). Consistently, Western blot of independently-derived

137 AALE isogenic lines demonstrated greater abundance of FOSL1 in KRAS- or RIT1-mutant cells

138 compared to wild-type expressing cells (Fig. 2b). TXNIP is an inhibitor of thioredoxin involved in

139 both redox regulation and glucose metabolism ${ }^{18,19}$. Prior literature identified HRAS ${ }^{\mathrm{G} 12 \mathrm{~V}_{\text {-induced }}}$

140 suppression of TXNIP transcription and protein translation ${ }^{20,21}$. TXNIP was among the top down-

141 regulated proteins in KRAS- and RIT1-mutant proteomes, and was decreased in Western blot 
142 analysis of independently derived cells (Fig. 2c). These validation data demonstrate the utility of

143 LC-MS/MS to describe protein expression changes and additionally suggest the mechanism of

144 RAS-mediated modulation of FOSL1 and TXNIP is shared with RIT1 ${ }^{\mathrm{M} 90 \mathrm{l}}$.

146 Next we compared the global effects of RIT1 ${ }^{\mathrm{WT}}$ and RIT1 ${ }^{\mathrm{M} 90 \mathrm{I}}$ to that of KRAS ${ }^{\mathrm{WT}}$ and KRAS

147 variants. Proteome and phosphoproteome data from RIT1 ${ }^{\mathrm{M} 90 \mathrm{I}}$-expressing cells were highly

148 correlated with $\mathrm{KRAS}^{\mathrm{G} 12 \mathrm{~V}}$ and $\mathrm{KRAS}^{\mathrm{Q61H}}$ profiles, suggesting largely similar downstream

149 consequences $(r=0.70-0.80$ and $0.72-0.75$ for proteome and phosphoproteome, respectively;

150 Fig. 2d). Despite differences in KRAS protein abundance, KRAS ${ }^{\mathrm{G} 12 \mathrm{~V}}$ and KRAS ${ }^{\mathrm{Q} 61 \mathrm{H}}$ proteomes

151 and phosphoproteomes were highly correlated (proteome $r=0.85$ and phospho $r=0.79$; Fig.

152 2d). In contrast, wild-type KRAS replicates were the most divergent of all profiles, showing 153 limited correlation to either the KRAS-mutant profiles or RIT1 profiles.

A recent study found that RIT1 variants, including M90I, may function by relieving negative regulation of RIT1 by a LZTR1-dependent proteasomal degradation mechanism ${ }^{11}$. Accordingly, overexpression of wild-type RIT1 should largely phenocopy expression of RIT1 ${ }^{\mathrm{M} 90 \mathrm{l}}$. Consistent with this idea, RIT1 ${ }^{\mathrm{WT}}$ cells more closely resembled both RIT1 ${ }^{\mathrm{M} 90 \mathrm{I}}$ and KRAS-mutant cells than KRAS $^{\text {WT }}$ cells (Fig. 2d). These data highlight a critical divergence between KRAS and RIT1: expression of wild-type KRAS is not capable of activating downstream oncogenic pathways, whereas expression of wild-type RIT1 in part resembles activation of RIT1 or KRAS by

162 mutation. We confirmed this observation in a principal component analysis of transcriptome 163 data, which further revealed a high degree of similarity between RIT1 ${ }^{\mathrm{WT}}$ and RIT1 ${ }^{\mathrm{M} 90 \mathrm{I}}$-regulated 164 gene expression (Fig. 2e and Supplementary Table 4). 


\section{Oncogenic RIT1 promotes epithelial-to-mesenchymal transition}

168 To identify the downstream pathways regulated by oncogenic KRAS and RIT1, we performed 169 gene set overlap analysis using MSigDB Hallmark Pathway gene $\operatorname{sets}^{22}$ (Fig. 3a). The epithelial-

170 to-mesenchymal transition (EMT) pathway was the most significant gene set enriched among 171 up-regulated proteins for both $\mathrm{KRAS}^{\mathrm{G} 12 \mathrm{~V}} / \mathrm{KRAS}^{\mathrm{Q} 61 \mathrm{H}}$ and $\mathrm{RIT} 1^{\mathrm{WT}} / \mathrm{RIT} 1^{\mathrm{M} 90 \mathrm{I}}$ cell lines. EMT is a

172 cellular transdifferentiation process promoted by cell-extrinsic signaling proteins and

173 orchestrated by activation of transcription factors such as Twist, Snail, and Zeb family

174 transcription factors ${ }^{23}$. It has long been observed that oncogenic RAS proteins, including KRAS

175 mutants, promote EMT. An EMT-signature is associated with KRAS dependence ${ }^{24}$, which has

176 been functionally linked to activation of FOSL $1^{25}$. Interestingly, we find both RIT1 ${ }^{\mathrm{M} 90 \mathrm{I}}$ and

$177 \mathrm{KRAS}^{\mathrm{G} 12 \mathrm{~V}} / \mathrm{KRAS}^{\mathrm{Q} 61 \mathrm{H}}$ are capable of promoting expression changes of key EMT markers,

178 including up-regulation of Vimentin, N-Cadherin, and Fibronectin, and downregulation of Keratin

17919 (Fig. 3b and Supplementary Fig. 2a). Although canonical EMT transcription factors Snail

180 (SNA1) and Slug (SNA2) were not detected by proteomic analysis, transcriptomes from RIT1-

181 and KRAS-mutant cells showed increased activity of these EMT transcription factors as

182 determined by ChEA3 transcription factor enrichment analysis (Fig. 3c-d and Supplementary

183 Fig. 2b). To our knowledge, this is the first demonstration of mutant RIT1 promoting EMT in any

184 cell type.

Oncogenic KRAS and RIT1 suppress Class I MHC expression via a post-transcriptional

187 mechanism

188 Among the top suppressed proteins with differential abundance in both mutant KRAS and $189 \mathrm{RIT}^{\mathrm{M} 90 \mathrm{I}}$ cells, were major histocompatibility complex $(\mathrm{MHC})$ proteins. Class I MHC proteins 190 HLA-A, HLA-B, HLA-C, and HLA-F were potently suppressed by KRAS ${ }^{\text {G12V }}$, KRAS ${ }^{\text {Q61H }}$, and

$191 \mathrm{RIT}^{\mathrm{M}}{ }^{\mathrm{9OI}}$ (Fig. 4a-b and Supplementary Fig. 3a). Recently there has been a renewed interest 
192 in expression of immune modulatory proteins in cancer due to the appreciation of the potent role

193 of the immune system in shaping cancer evolution. Further understanding the regulation of HLA

194 expression in cancer is particularly critical in metastatic KRAS-mutant lung adenocarcinoma,

195 where chemotherapy combined with immune checkpoint blockade is often used in the first-line

196 setting.

Class I MHC genes HLA-A, HLA-B, and HLA-C harbor loss-of-function mutations in cancer ${ }^{26}$,

199 demonstrating selective pressure to lose MHC function during tumorigenesis. Both MHC

200 expression loss and upregulation of the immune suppressive protein PD-L1 enable tumor

201 evasion of T-cell recognition of aberrant cancer cell proteins ${ }^{27}$. Moreover, expression loss of

202 HLA proteins or B2M, another MHC Class I complex protein, is associated with resistance to

203 immunotherapy in cancer ${ }^{28}$. We found that RIT1 ${ }^{\mathrm{M} 901}, \mathrm{KRAS}^{\mathrm{G} 12 \mathrm{~V}}$, and KRAS ${ }^{\mathrm{Q} 61 \mathrm{H}}$ cells all promoted

204 loss of B2M protein abundance in addition to HLA protein loss (Fig. 4c).

206 Class I MHC expression is known to be dynamically regulated by upstream signals controlled by 207 interferon gamma exposure, NF-kB signaling, and chromatin regulators such as $\mathrm{EZH} 2^{29,30}$. Each 208 of these mechanisms involves transcriptional regulation of class I MHC genes. However, there 209 were no transcriptional differences in HLA genes in the KRAS-mutant and RIT1-mutant cells nor 210 were any transcriptional differences observed in the upstream regulators of MHC Class I

211 expression NLRC5 and IRF1 and IRF2 (Supplementary Fig. 3b). Moreover, we excluded the

212 possibility that lentiviral transduction or expression of a foreign antigen was responsible for the

213 HLA suppression, because HLA protein expression was maintained or enhanced in RIT1 ${ }^{\text {WT. }}$

214 expressing cells as well as vector control cells, which express the Renilla luciferase gene. 
216 To identify the possible mechanism of RIT1 ${ }^{\text {M90I }}$ - and KRAS-mediated MHC suppression, we

217 identified other proteins that, like HLA, were upregulated in RIT1 ${ }^{\text {WT }}$ cells but suppressed in

218 RIT1-mutant and KRAS-mutant cells (Fig. 4d). This analysis revealed the pervasive

219 downregulation of the Rab-mediated ER/Golgi vesicle-trafficking pathway that controls MHC

220 Class I processing and presentation as well as the MHC Class I complex proteins themselves

221 (Fig. 4e). In addition, expression of the proteasomal subunit PSMB9 correlated with loss of the

222 MHC processing machinery (Fig. 4f and Supplementary Fig. 3c). Loss of PSMB9, also known

223 as LMP2, has been previously linked to loss of MHC expression after oncogenic

224 transformation ${ }^{31}$. We conclude that $\mathrm{RIT}^{\mathrm{M} 90 \mathrm{I}}$ and KRAS ${ }^{\mathrm{G} 12 \mathrm{~V}} / \mathrm{KRAS}^{\mathrm{Q} 61 \mathrm{H}}$ suppress MHC Class I

225 expression through a post-transcriptional mechanism possibly involving PSMB9. Further

226 investigation of MHC Class I expression loss driven by these oncogenic RIT1 and KRAS is

227 critical to better understand the role of RAS and RIT1 signaling on immune evasion in cancer.

229 The identification of a major class of proteins regulated at the post-transcriptional level in RIT1-

230 and KRAS-transformed lung epithelial cells brought to our attention the possibility of other post-

231 transcriptional regulation by RIT1 and KRAS. Indeed, oncogenic RAS signaling profoundly

232 alters cap-dependent translation via activation of the p90 ribosomal S6 kinases (RSKs) ${ }^{32}$ and

$233 \mathrm{PI} 3 \mathrm{~K} / \mathrm{mTOR}^{33}$, so differential protein translation could significantly contribute to altered protein

234 abundance in RAS-transformed cells. To determine whether there were other protein classes in

235 addition to MHC Class I proteins with significant post-transcriptional regulation, we performed a

236 global correlation analysis of the transcriptome and proteome. Significant linear correlations

237 between transcript and protein abundance were observed for RIT1 and KRAS variants, with the

238 correlation highest for cells expressing mutant $\mathrm{KRAS}^{\mathrm{G} 12 \mathrm{~V}}(r=0.3725)$ or $\mathrm{KRAS}^{\mathrm{Q} 1 \mathrm{H}}(r=0.3620)$

239 (Fig. 4g). While expression of the majority of genes were correlated at the RNA and protein

240 levels, the metallothionein protein family including MT1E, MT1F and MT1X was highly 
241 upregulated in the proteome but not transcriptome of KRAS-mutant cells (Fig. $\mathbf{4 g}$ ). In addition,

242 intermediate filament proteins were also substantially regulated post-transcriptionally; both

243 alpha-internexin (INA) and vimentin (VIM) were expressed more highly in the proteome than

244 expected from RNA-seq data (Fig. 4g). These data highlight the utility of LC-MS/MS to identify

245 protein abundance changes that would not be predicted from transcriptome analysis.

Phosphoproteome profiling illuminates shared and unique signaling by RIT1 and KRAS

248 Protein phosphorylation is a reversible and dynamic mechanism of intracellular signaling that enables rapid intracellular transduction of signals controlling cell proliferation, survival, and metabolism. Although both RIT1 and KRAS act as GTPase switches, they both stimulate activation of cellular protein kinases such as BRAF. We therefore evaluated protein phosphorylation regulated by wild-type and mutant RIT1 and KRAS. Phosphosite abundance was expressed as a relative abundance normalized to the total protein abundance for each

254 phosphoprotein. Unsupervised hierarchical clustering of the phospho-signatures identified the $255 \mathrm{RIT}^{\mathrm{M} 90 \mathrm{O}}$ phosphoproteome as most similar to KRAS ${ }^{\mathrm{G} 12 \mathrm{~V}}$ and KRAS ${ }^{\mathrm{Q} 61 \mathrm{H}}$ phospho-signatures

256 (Supplementary Fig. 4a). We performed Kinase-Substrate Enrichment Analysis (KSEA ${ }^{34}$ ), 257 which uses kinase-substrate pairings from PhosphoSitePlus ${ }^{35}$ and NetworkIN ${ }^{36}$ to identify 258 differential phosphorylation of kinase-substrate families (Supplementary Table 5). These data

259 further confirmed the similarity in phosphorylation state between RIT1-mutant and KRAS-mutant 260 cells. The top kinases with increased substrate phosphorylation in RIT1-mutant and KRAS-

261 mutant cells were ribosomal S6 kinase (RPS6KA1), Protein kinase C (PRKCA), AKT1, and

262 MAPKAPK2 (Fig. 5a-c, Supplementary Fig. 4b-e, and Supplementary Table 5). The levels of 263 phosphorylation of RPS6KA1 and MAPKAPK2 substrates were enhanced most strongly in the 264 mutant cells and less in RIT1 WT and KRAS WT-expressing cells (Fig. 5b-c). Substrates of 265 Aurora kinase B and CDK1 and PAK1 were suppressed in RIT1- and KRAS-mutant cells (Fig. 
266 5a and Supplementary Fig. 4b-c and Supplementary Fig. 4f). Although the total

267 phosphorylation of each substrate reflects the balance between kinases and phosphatases in

268 the cell, these data suggest that $\mathrm{RIT}^{\mathrm{M} 90 \mathrm{l}}$, like oncogenic KRAS, can activate the canonical RAS

269 effector pathways involving S6 kinase and AKT.

271 Next we assessed the divergent functions of RIT1 ${ }^{\mathrm{M} 90 \mathrm{I}}$ and KRAS $\mathrm{K}^{\mathrm{G} 12 \mathrm{~V}} / \mathrm{KRAS}^{\mathrm{Q} 61 \mathrm{H}}$ by identifying

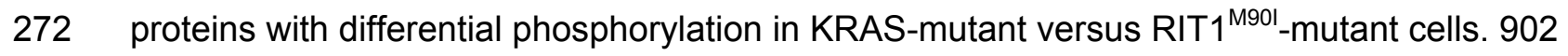

273 differentially phosphorylated sites were identified by two-tailed t-test and multiple hypothesis

274 correction (Fig. 5d; FDR < 0.05). Interestingly, the top site with lower phosphorylation in

$275 \mathrm{KRAS}^{\mathrm{G} 12 \mathrm{~V}}$ and KRAS ${ }^{\mathrm{Q61H}}$ cells was EGFR serine 1026 (Fig. 5d). In lung adenocarcinoma, KRAS

276 mutations and EGFR mutations are mutually exclusive, suggesting a powerful genetic

277 interaction between these two genes. Recent work demonstrated that mutant KRAS and EGFR

278 display synthetic lethality ${ }^{37}$. However the mechanism underlying this lethality is unknown.

279 Further inspection of the phospho-proteome signatures revealed extensive alteration of EGFR

280 phosphorylation by $\mathrm{KRAS}^{\mathrm{G} 12 \mathrm{~V}}$ and $\mathrm{KRAS}^{\mathrm{Q61H}}$, but not by RIT1 ${ }^{\mathrm{M} 90 \mathrm{I}} .11$ of $12 \mathrm{EGFR}$ sites detected

281 by LC-MS/MS occur in the cytoplasmic carboxy-terminal tail of EGFR (Fig. 5e). Five of these

282 sites (S991, S991/T993 double phosphorylation, S1026, S1039, and T1041/S1045 double

283 phosphorylation) were significantly depleted of phosphorylation in KRAS ${ }^{\mathrm{G} 12 \mathrm{~V}}$ and KRAS ${ }^{\mathrm{Q61H}}$ -

284 expressing cells but not in $\mathrm{RIT}^{\mathrm{M} 90 \mathrm{I}}$-expressing cells. Interestingly, these sites lie in a region of

285 EGFR that is involved in receptor internalization and endocytosis ${ }^{38}$ and a phosphorylation-

286 deficient mutant at $\mathrm{S} 991$ is defective at internalization ${ }^{39}$. Consistently, EGFR protein abundance

287 was increased in KRAS-mutant cells (Supplementary Fig. 4g) Although the specific regulatory

288 mechanisms leading to this depletion remain unknown, these data point to the existence of

289 feedback regulatory signaling from oncogenic KRAS to EGFR. 
291 Examining phosphorylation uniquely promoted by $\mathrm{KRAS}^{\mathrm{G} 12 \mathrm{~V}}$ and $\mathrm{KRAS}^{\mathrm{Q} 61 \mathrm{H}}$, we identified USO1

292 phosphorylation at S48 as one of the top most significantly increased phosphorylation events in

293 KRAS-mutant cells. USO1, also known as p115, is a vesicle tethering factor involved in ER-

294 Golgi intracellular trafficking ${ }^{40}$. Although wild-type KRAS and KRAS-mutant proteomic

295 signatures were largely divergent, USO1 serine 48 phosphorylation was promoted by both

$296 \mathrm{KRAS}^{\mathrm{WT}}$ and mutant KRAS (Fig. 5f). KRAS relies on vesicle trafficking to ensure proper post-

297 translational farnesylation and palmitoylation, which are required for targeting of KRAS to the

298 plasma membrane ${ }^{41}$. We hypothesized that USO1 S48 phosphorylation was therefore

299 correlated with KRAS expression rather than activity. Indeed, a significant correlation was

300 observed between overall KRAS expression and USO1 phosphorylation (Fig. $\mathbf{5 g}$ ). In contrast,

301 USO1 S48 phosphorylation was only modestly changed in RIT1-mutant cells (Fig. 5d). Notably,

302 RIT1 lacks the farnesylation and palmitoylation signals present in RAS isoforms ${ }^{42}$, so the

303 differential regulation of USO1 by KRAS and RIT1 may be related to differences in RIT1 and

304 KRAS trafficking.

Also among the top differentially phosphorylated sites were 32 phosphorylation sites in AHNAK

proteins 1 and 2. AHNAK and AHNAK2 are large scaffolding proteins that have been implicated as tumor suppressor proteins in breast and lung cancer ${ }^{43,44}$. Among all phospho-proteins, a

309 higher proportion (32/117) of sites on AHNAK and AHNAK2 were differentially phosphorylated

310 than expected by chance $(P<0.0001$ by Chi Square test; Supplementary Fig. 4h). Intriguingly,

311 two recent proximity-labeling proteomic studies identified AHNAK and AHNAK2 as KRAS-

312 interacting proteins ${ }^{45,46}$, raising the possibility that a direct physical interaction between KRAS

313 and AHNAK proteins may be involved in the differential AHNAK phosphorylation we observe. 


\section{Discussion}

Here we describe quantitative proteomic, phosphoproteomic, and transcriptomic datasets that

318 provide the first systematic view of the RIT1 ${ }^{\mathrm{M} 90 \mathrm{I}}$-regulated signaling network. These datasets

319 were generated from isogenic human lung epithelial cells to provide a physiological view of the

320 consequences of RIT1 activation in the same cellular compartment that is involved in lung

321 adenocarcinoma, a tumor type with prevalent mutations in KRAS and RIT1. Broadly, we find

322 that 'omic signatures from RIT1 ${ }^{\mathrm{M} 90 \mathrm{I}}$-expressing cells largely phenocopy those from cells with

323 overexpression of wild-type RIT1. This finding lends further support to the notion that oncogenic

324 RIT1 variants function at least in part through increasing RIT1 abundance ${ }^{11}$. This is in contrast

325 to KRAS, where overexpression of wild-type KRAS induces signatures unrelated or opposite to

326 that of oncogenic KRAS variants G12V and Q61H. The opposing functions of wild-type and

327 mutant KRAS is consistent with recent evidence suggesting that KRAS functions as a dimer and

328 that wild-type KRAS directly inhibits the function of oncogenic KRAS variants via physical

329 dimerization ${ }^{47}$. This divergence in the function of wild-type RIT1 and KRAS hints at fundamental

330 differences in molecular regulation of each wild-type GTPase. The ability of RIT1 to promote

331 downstream RAF/MEK/ERK signaling when aberrantly expressed suggests that RIT1 may not

332 be subject to the same tight regulation by GTPase-activating proteins (GAPs) that normally

333 keep RAS in an inactive state. Furthermore, these data raise the possibility that wild-type RIT1

334 overexpression in RIT1-amplified cancers may contribute to tumorigenesis. RIT1, on

335 chromosome 1q, is frequently amplified in uterine carcinosarcoma, liver hepatocellular cancer,

336 cholangiocarcinoma, breast cancer, lung adenocarcinoma, and ovarian cancer. RIT1 mRNA

337 expression is increased in amplified cases, regardless of tissue type, raising the possibility that

338 RIT1 overexpression could play a role in tumorigenesis in these cancers. 
340 We find that RIT1 ${ }^{\mathrm{M} 90 \mathrm{I}}, \mathrm{KRAS}^{\mathrm{G} 12 \mathrm{~V}}$, and KRAS ${ }^{\mathrm{Q61H}}$ share the ability to activate canonical RAS

341 effector pathways PI3K/AKT and RAF/MEK. Likely as a consequence of RAF/MEK signaling to

342 FOSL1, RIT1 ${ }^{\mathrm{M} 90 \mathrm{I}}$ also shares the ability to induce EMT markers including Vimentin, N-cadherin,

343 and fibronectin. KRAS and RIT1 variants also shared the ability to profoundly suppress HLA-A, -

$344 \mathrm{~B}$, and $-\mathrm{C}$ expression via a posttranscriptional mechanism. Taking advantage of differential

345 expression of HLA proteins between RIT1 ${ }^{\text {WT }}$ and all other isogenic lines, we identified an entire

346 Rab-mediated endocytic network that was lost together with HLA proteins in RIT1- and KRAS-

347 mutant cells. This downregulated module also included PSMB9, a subunit of the

348 immunoproteasome that is involved in antigen processing for class I MHC presentation. RAS

349 oncogenes have long been recognized to suppress surface MHC expression ${ }^{48}$, in some cases

350 transcriptionally and in others post-transcriptionally ${ }^{31}$. Our data link both oncogenic RIT1 and

351 RAS to modulation of the processing and trafficking of MHC Class I molecules. Further

352 identification of the mechanism of RIT1/RAS-mediated MHC suppression will provide a better

353 understanding of tumor immune evasion which is critically needed to optimize patient

354 stratification of cancer immunotherapy.

356 In addition to the largely concordant regulation of proteins by mutant RIT1 and KRAS, we

357 identified several unique phosphoproteins with differential abundance in RIT1 ${ }^{\mathrm{M} 901}$ and KRAS-

358 mutant cells. These included EGFR, a key oncoprotein in lung adenocarcinoma, which showed

359 reduced phosphorylation of sites involved in receptor internalization and endocytic trafficking.

360 Given the potent genetic interactions between KRAS and EGFR in lung cancer and colon

361 cancer, it is attractive to speculate that feedback regulation of KRAS to EGFR could provide an

362 explanatory mechanism for this phenomenon. Future work is needed to determine the basis of

363 the specific regulation of EGFR phosphorylation by oncogenic KRAS but not RIT1. 
364 Together, these results demonstrate the power of quantitative proteomics and transcriptomics to

365 provide global views of cancer oncogene signaling. Our multi-omic analysis validated known

366 consequences of RAS activation such as EMT and activation of RAF/MEK and PI3K signaling.

367 For the first time, we gained a global view of RIT1 function, which confirmed its ability to

368 stimulate canonical RAS signaling. However, phosphoproteomic profiling identified a number of

369 key divergent mechanisms between KRAS- and RIT1-mutant cells, which point to the existence

370 of novel, unique regulators or effectors of KRAS and RIT1 still to be identified. Future work is

371 needed to investigate the mechanisms of these differences between KRAS and RIT1, the

372 results of which will have important implications for cancer therapy and Noonan Syndrome.

373

374

375

376 


\section{Methods}

\section{Isogenic Cell Line Generation}

381 Plasmid constructs were cloned using Gateway Technology (Invitrogen/ThermoFisher) using

382 pLX301 destination vector (Broad Institute) and pDONR223-RIT1 donor vectors previously

383 described ${ }^{5}$. Lentivirus was generated by transfection of HEK293T cells with packaging and

384 envelope vectors using standard protocols. AALE cells were a kind gift of Jesse Boehm (Broad

385 Institute). Isogenic cells were generated by transduction of lentivirus generated from pLX317-

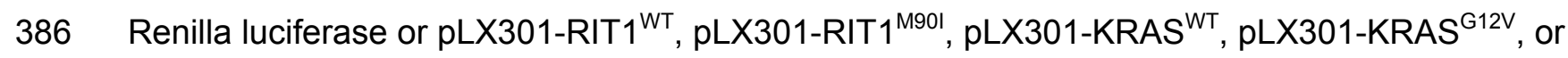

$387 \mathrm{pLX} 301-\mathrm{KRAS}^{\mathrm{Q} 61 \mathrm{H}}$ and selection with puromycin. Stable pools of cells were maintained in small

388 airway growth medium (Lonza).

Soft Agar Assay

$3911 \times 10^{5}$ cells were suspended in $1 \mathrm{ml}$ of $0.33 \%$ select agar in small airway growth medium without 392 EGF (Lonza) and plated on a bottom layer of $0.5 \%$ select agar in the same media in six-well 393 dishes. Each cell line was analyzed in triplicate. Colonies were photographed after 14-21 days 394 and quantified using CellProfiler ${ }^{49}$.

\section{Transcriptome profiling}

397 Three technical replicates per cell line were harvested at $\sim 90 \%$ confluence $(n=18$ total dishes).

398 Cells were lysed and total RNA was extracted using Direct-zol RNA Miniprep plus (Zymo

399 Research). Libraries were constructed using the non-strand-specific poly-A selection Illumina

400 TruSeq kit for 50bp paired-end reads. Libraries were then pooled and sequenced on the

401 Illumina NovaSeq platform (Fred Hutch Genomics Core). Reads were aligned to the human

402 reference genome build hg19/GRCh37 using STAR v.2.5.3 $\mathrm{a}^{50}$. Alignments were annotated for

403 duplicates and read groups, and then reordered and indexed, using Picard Tools v.1.114 ${ }^{51}$. 
404 Read statistics for each RNA-seq sample were calculated using RSeQC ${ }^{52}$. Quantification of

405 gene transcripts was performed by the featureCounts program within the Subread package ${ }^{53}$,

406 using hg19 gene annotation from UCSC Gene level CPM and RPKM values were calculated

407 with edgeR v.3.22. $3^{54}$, and converted into transcripts per million (TPM values with an in-house

408 script. In total, 12,887 genes were identified with average logCPM at least 0.1 across all

409 samples. Differential expression analyses comparing KRAS or RIT1 perturbed cell lines against

410 vector control lines were performed using edge ${ }^{54}$.

High performance liquid chromatography tandem mass spectrometry (LC-MS/MS)

413 Cells were washed in ice-cold PBS, scraped into PBS, pelleted, and snap frozen in liquid

414 nitrogen. The experimental workflow for sample processing, TMT-labeling, peptide enrichment,

415 and LC-MS/MS were largely as previously described ${ }^{55}$. Briefly, pellets were lysed in $200 \mu$ of

416 chilled urea lysis buffer (8 M urea, $75 \mathrm{mM} \mathrm{NaCl}, 50 \mathrm{mM}$ Tris (pH 8.0), $1 \mathrm{mM}$ EDTA, $2 \mu \mathrm{g} / \mathrm{ml}$

417 aprotinin, 10 mg/ml leupeptin, 1 mM PMSF, 1:100 (vol/vol) Phosphatase Inhibitor Cocktail 2,

418 1:100 Phosphatase Inhibitor Cocktail 3, $10 \mathrm{mM} \mathrm{NaF}$, and $20 \mu \mathrm{M}$ PUGNAc) for each 50 mg

419 portion of wet-weight tissue. Lysates were reduced with 5mM DTT, alkylated with 10 mM IAM,

420 and digestion performed in solution with $1 \mathrm{mAU}$ LysC per $50 \mu \mathrm{g}$ of total protein and trypsin at an

421 enzyme/substrate ratio of 1:49. Reactions were quenched with $\mathrm{FA}$ and brought to $\mathrm{pH}=3$ with

422 FA. Peptides were desalted on $200 \mathrm{mg} \mathrm{tC} 18$ SepPak cartridges and dried by vacuum

423 centrifugation. $340 \mu \mathrm{g}$ of peptides were labeled with 10-plex Tandem Mass Tag reagents

424 (TMT10, Fisher Scientific), according to manufacturer's instructions. To enable quantification of

425 peptides across all 12 samples, the samples were labeled in sets of 10 across two different

426 TMT10 pools in a crossover design with 8 of 12 samples analyzed in both TMT10 pools. A

$42750 / 50$ mix of both AALE vector control lysates was used as an internal reference in both TMT10

428 runs (Supplementary Fig. 1b). 
430 Each TMT10-plex was desalted in a $200 \mathrm{mg} \mathrm{tC18} \mathrm{SepPak} \mathrm{cartridge} \mathrm{and} \mathrm{fractionated} \mathrm{using}$

431 offline HPLC. 5\% of each fraction was collected into an HPLC vial for proteome analysis by LC-

432 MS/MS. The remaining 95\% was processed for phospho-peptide enrichment via immobilized

433 metal affinity chromatography (IMAC). IMAC enrichment was performed using Ni-NTA

434 Superflow Agarose beads incubated with peptides solubilized in a final concentration of $80 \%$

435 MeCN/0.1\% TFA. Phospho-enriched peptides were desalted and collected into an HPLC vial for 436 analysis by LC-MS/MS.

438 Online fractionation was performed using a nanoflow Proxeon EASY-nLC 1200 UHPLC system

439 (Thermo Fisher Scientific) and separated peptides were analyzed on a benchtop Orbitrap Q

440 Exactive Plus mass spectrometer (Thermo Fisher Scientific) equipped with a nanoflow

441 ionization source (James A. Hill Instrument Services, Arlington, MA). In-house packed columns

442 (20 cm x $75 \mu \mathrm{m}$ diameter C18 silica picofrit capillary column; $1.9 \mu \mathrm{m}$ Reprosll-Pur C18-AQ

443 beads, Dr. Maisch GmbH, r119.aq; Picofrit $10 \mu \mathrm{m}$ tip opening, New Objective, PF360-75-10-N-

444 5). Mobile phase flow rate was $200 \mathrm{~nL} / \mathrm{min}$, comprised of $3 \%$ acetonitrile $/ 0.1 \%$ formic acid

445 (Solvent A) and $90 \%$ acetonitrile $/ 0.1 \%$ formic acid (Solvent B). The 110 min LC-MS/MS

446 method consisted of a 10 min column-equilibration procedure; a 20 min sample-loading

447 procedure; and the following gradient profile: (min: \% B) 0:2; 2:6; 85:30; 94:60; 95:90; 100:90;

$448101: 50 ; 110: 50$ (the last two steps at $500 \mathrm{~nL} / \mathrm{min}$ flow rate). Data-dependent acquisition was

449 performed using Xcalibur QExactive v2.4 software in positive ion mode at a spray voltage of

$4502.00 \mathrm{kV}$. MS1 Spectra were measured with a resolution of 70,000, an AGC target of $3 \mathrm{e} 6$ and a

451 mass range from 300 to $1800 \mathrm{~m} / \mathrm{z}$. Up to $12 \mathrm{MS} / \mathrm{MS}$ spectra per duty cycle were triggered at a

452 resolution of 35,000 , an $A G C$ target of $5 \mathrm{e} 4$, an isolation window of $0.7 \mathrm{~m} / \mathrm{z}$, a maximum ion time

453 of $120 \mathrm{msec}$, and normalized collision energy of 30. Peptides that triggered MS/MS scans were 
454 dynamically excluded from further MS/MS scans for $20 \mathrm{sec}$. Charge state screening was

455 enabled to reject precursor charge states that were unassigned, 1 , or $>6$. Peptide match was

456 set to preferred for monoisotopic precursor mass assignment.

458 Protein-peptide identification, phosphosite localization, and quantification

459 MS data was interpreted using the Spectrum Mill software package v6.0 pre-release (Agilent

460 Technologies, Santa Clara, CA. MS/MS spectra were merged if they were acquired within +/- 45

$461 \mathrm{sec}$ of each other with the same precursor $\mathrm{m} / \mathrm{z}$. Also, MS/MS spectra that did not have a

462 sequence tag length $>0$ (i.e., minimum of two masses separated by the in chain mass of an

463 amino acid) or did not have a precursor $\mathrm{MH}+$ in the range of $750-6000$ were excluded from

464 searching. MS/MS spectra searches were performed against a concatenated UniProt human

465 reference proteome sequence database containing 58,929 human proteins including isoforms

466 (obtained 10/17/2014) and 150 additional common laboratory contaminants. ESI-QEXACTIVE-

467 HCD-3 scoring parameters were used for both whole proteome and phosphoproteome datasets.

468 Spectra were allowed +/- 20 ppm mass tolerance for precursor as well as product ions, 30\%

469 minimum matched peak intensity, and "trypsin allow P" was set as enzyme specificity with up to

4704 missed cleavages allowed. Carbamidomethylation at cysteine was set as fixed modification

471 together with TMT10 isobaric labels at lysine residues (N-termini would be considered

472 regardless if it was TMT labelled). Acetylation of protein $\mathrm{N}$-termini andoxidized methionine were

473 set as variable modifications with a precursor $\mathrm{MH}+$ shift range of -18 to $64 \mathrm{Da}$ for the proteome

474 searches. For the phosphoproteome searches the precursor $\mathrm{MH}+$ shift range was set to 0 to

$475272 \mathrm{Da}$ and variable modifications of phosphorylation of serine, threonine, and tyrosine.

476 Identities interpreted for individual spectra were automatically designated as confidently

477 assigned using the Spectrum Mill autovalidation module to use target-decoy based false

478 discovery rate (FDR) estimates to apply score threshold criteria. For the whole proteome 
479 datasets, thresholding was done at the spectral $(<1.2 \%)$ and protein levels $(<0.1 \%)$. For the

480 phosphoproteome datasets, thresholding was done at the spectral $(<1.2 \%)$ and phosphosite

481 levels $(<1.0 \%)$

482 Replicates across TMT-plexes were highly correlated (Supplementary Fig. 1c) with median

483 Pearson $r=0.87$ and 0.69 for proteome and phosphoproteome, respectively. Technical

484 replicates and biological replicates were merged to generate final total proteome and phospho-

485 proteome profiles for each isogenic cell line (Supplementary Tables 1 and 2). Replicate-level

486 profiles are also supplied as JavaScript Object Notation (.json) files that can be visualized and

487 analyzed using the Morpheus Matrix Visualization and Analysis Software at

488 https://software.broadinstitute.org/morpheus (Supplementary Files 1 and 2). Differential

489 protein and phospho-site signatures were generated by computing the mean $\log _{2}$ (fold change)

490 of the abundance of each site in each sample compared to the vector control cells. Statistical

491 significance of differentially abundant proteins and phosphosites was determined by performing

492 a one sample moderated t-test with multiple hypothesis correction (Supplementary Tables 1

493 and 2).

494

495 Integrative Analysis

496 Correlation of changes in protein expression and changes in RNA expression was modeled

497 using R's Im() function. 95\% prediction intervals were calculated to determine genes with weak

498 concordance between protein and RNA expression.

500 Gene Set Enrichment Analysis

501 Analysis of enrichment of KRAS signaling in differential RNA expression profiles was performed

502 in R with the goseq package ${ }^{56}$. KRAS signaling gene sets were taken from MSigDB hallmark

503 gene sets $22,57$. 
505 Transcription Factor Target Enrichment Analysis

506 Analysis of over-representation of Transcription Factor targets was performed with ChEA3 by

507 submitting lists of differentially expressed genes $(|\mathrm{LFC}|>1$ and FDR $<0.05)$. ChEA3 performs

508 Fisher's Exact Test to compare the input gene set to TF target gene sets in six different

509 libraries $^{58}$. Analysis of the Enrichr Queries library was selected as the focus of the present

510 study. Transcription factors resulting from this analysis were annotated as one of four groups of

511 EMT association. These four groups were the Snail gene family, confirmed EMT genes defined

512 by $\mathrm{dbEMT}^{59}$, genes shown to be associated with EMT in at least one study in literature, and

513 genes unrelated to EMT.

515 Antibodies and immunoblotting

516 Antibodies against FOSL1 (D80B4), TXNIP (D5F3E), and Vimentin (D21H3) were purchased

517 from Cell Signaling Technology. Vinculin (V9264) was purchased from Sigma Aldrich.

518 Secondary antibodies StarBright Blue 700 Goat anti-Rabbit lgG, StarBright Blue 520 Goat anti-

519 Rabbit IgG and StarBright Blue 520 Goat anti-Mouse IgG (12005867) were purchased from Bio-

520 Rad. Antibody against RIT1 (\#53720) was purchased from Abcam. Cell lysates were prepared

521 in RTK lysis buffer with protease (11836153001, Roche) and phosphatase (04906837001,

522 Roche) inhibitors added and quantified by the BCA assay (Thermo Scientific Waltham, MA).

523 Samples were then boiled in Laemmli buffer (1610747, Bio-Rad, Hercules, CA) and 50 ug of

524 protein was loaded onto 4-15\% Mini-Protean TGX (4561084, Bio-Rad, Hercules, CA) gels.

525 Protein gels were run and transferred to PVDF membranes (1704274, Bio-Rad, Hercules, CA)

526 according to manufacturer's instructions. Proteins were detected by specific primary antibody

527 and secondary antibody then visualized using the ChemiDoc MP Imaging System (Bio-Rad,

528 Hercules, CA). 


\section{KSEA analysis}

532 Kinase-substrate enrichment analysis (KSEA) ${ }^{60}$ was performed using the KSEA App ${ }^{34}$

533 (https://casecpb.shinyapps.io/ksea/) using kinase-substrate mappings from PhosphoSitePlus ${ }^{35}$

534 and a $p$ value threshold of $<0.05$. A minimum of five detected phospho-site substrates were

535 needed for kinases to be included in the analysis. The full list of kinase scores and number of

536 substrates are shown in Supplementary Table 5. 36 kinases had sufficient substrate sites

537 detected to be included in the analysis. Kinase-substrate mappings are shown in

538 Supplementary Table 5.

DATA AVAILABILITY

541 The RNA-seq data have been deposited in the NCBI Gene Expression Omnibus database with

542 accession number GSE146479. All mass spectra contributing to this study can be downloaded

543 in the original instrument vendor format from the MassIVE online repository (Accession number

544 to be updated prior to publication.)

\section{ACKNOWLEDGEMENTS}

547 We thank Drs. Athea Vichas and Jon Cooper (Fred Hutchinson Cancer Research Center) for

548 advice, discussion, and critical reading of the manuscript. We thank Dr. D.R. Mani (Broad

549 Institute) for guidance on statistical analysis. This research was funded in part through the

550 National Cancer Institute (NCI) K99/R00 CA197762 to AHB, NIH/NCl Cancer Center Support

551 Grant P30 CA015704, NCI Clinical Proteomic Tumor Analysis Consortium grants NIH/NCI U24-

552 CA210986 and NIH/NCI U01 CA214125 to SAC. AL was supported in part by NSF IGERT

553 DGE-1258485. KH was supported in part by PHS NRSA T32GM007270 from NIGMS. 


\section{AUTHOR CONTRIBUTIONS}

556 A.H.B. conceived of and directed the study. S.C. and P.M. supervised the LC-MS/MS

557 experiments. F.M. performed the proteomics data analysis, with contributions from

558 A.H.B. and K.H. A.L. performed the transcriptome analysis. A.L. and A.H.B. wrote the

559 manuscript. A.L., K.H., S.M., I.F., S.F., J.W., and A.H.B. performed experiments. All

560 authors discussed results and provided input on the manuscript. 


\section{Figure Legends}

566 Figure 1. Comparative multi-omic profiling of KRAS- and RIT1-mutant human lung

567 epithelial cells. a, Protein alignment of KRAS-4B (Uniprot \#P01116-2) and RIT1 Isoform 1

568 (Uniprot \#Q92963-1) generated by ClustalW2 ${ }^{61}$. Stars indicate the position of the RIT1 ${ }^{\text {M90I }}$ or

$569 \mathrm{KRAS}^{\mathrm{G} 12 \mathrm{~V}}$ and KRAS ${ }^{\mathrm{Q} 61}$ variants used in this study. Asterisks indicate fully conserved residues.

570 Colons indicate strongly conserved residues. Periods indicate weakly conserved residues. b,

571 Soft agar colony formation assay of isogenic AALE human lung epithelial cells. ${ }^{* *}, \mathrm{p}<0.01 ;{ }^{* * * *}$,

$572 p<0.0001$ by two-tailed t-test. c, Western blot using anti-RAS and anti-RIT1 antibodies (top

573 panels), or antibodies against phosphorylated ERK1/2 or vinculin (loading control). SE $=$ short

574 exposure, LE = long exposure. Isogenic AALE cells were cultured in the presence or absence of

575 EGF for 12 hours. d, LC-MS/MS workflow for generation of proteome and phosphoproteome

576 profiles. bRP, basic reverse phase chromatography. IMAC, immobilized metal affinity

577 chromatography. e, Workflow for Illumina RNA-seq analysis. f, mRNA quantification in

578 transcripts per million (TPM) showing mean \pm SD of RIT1 (left) or KRAS (right) in isogenic AALE

579 cells, $n=3$ per cell line. *,$p<0.05 ;{ }^{* * * *}, p<0.0001$ by two-tailed Student's t-test compared to

580 vector control cells. g, RNA-seq quantification of variant allele expression. Data shown is the

581 percentage of reads at the $\mathrm{M} 90 \mathrm{I}, \mathrm{G} 12 \mathrm{~V}$, or $\mathrm{Q} 61 \mathrm{H}$ variant site for the variant allele or wild-type

582 allele.

583 Figure 2. Quantitative proteome and transcriptome profiling identifies similarity in

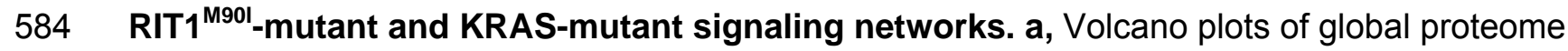

585 data from isogenic AALE cells showing the $\log _{2}$ (fold change) (“LFC") in protein abundance in

586 each cell line compared to vector control cells. The y-axis displays the negative $\log _{10}(p$ value $)$

587 calculated from a one sample moderated t-test with multiple hypothesis correction by the

588 Benjamini-Hochberg method. b, Western blot validation of FOSL1 increased protein abundance

589 in RIT1- and KRAS-mutant cells. The chart shows the LFC of FOSL1 as determined by LC- 
590 MS/MS. Western blot below shows FOSL1 abundance or Vinculin (loading control). c, Western

591 blot validation of TXNIP protein abundance in RIT1- and KRAS-mutant cells. The chart shows

592 the LFC of TXNIP as determined by LC-MS/MS. d, Correlation heatmap showing pairwise

593 Pearson and Spearman correlations of each proteome and phosphoproteome replicate to every

594 other replicate. To enable correlation of proteome with phosphoproteome, phosphosites were

595 collapsed to the protein level by taking the median of all phosphosites for each protein. e,

596 Principal component analysis (PCA) of RNA-seq data. Circles correspond to control vector or

597 wild-type replicates. Diamonds correspond to RIT1- or KRAS-mutant profiles.

598 Figure 3. RIT1 ${ }^{\text {M90I }}$ promotes epithelial-to-mesenchymal (EMT) transition. a, Gene set

599 overlap analysis of up-regulated (“Up”; LFC>2) and down-regulated (“down”; LFC<-2) proteins

600 using MSigDB Hallmark Pathways ${ }^{22}$. "K" and "R" indicate analysis based on mean LFC of

$601 \mathrm{KRAS}^{\mathrm{G} 12 \mathrm{~V}} / \mathrm{KRAS}^{\mathrm{Q} 61 \mathrm{H}}$ cells or RIT1 ${ }^{\mathrm{WT}} / \mathrm{RIT}^{1 \mathrm{M} 90 \mathrm{I}}$ cells, respectively. Circle size corresponds to the $p$

602 value of gene set overlap analysis determined by MSigDB. b, LFC of protein abundance of EMT

603 marker genes as determined by LC-MS/MS, relative to vector control cells. c, Transcription

604 factor target enrichment analysis of differentially expressed genes in RIT1 ${ }^{\text {M90I }}$-mutant cells using

605 Enrichr libraries. FET, Fisher's exact test. Red = Snail family. Orange = confirmed EMT genes in

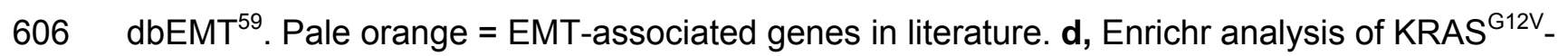

607 mutant proteome data. Annotation is the same as in c.

Figure 4. RIT1- and KRAS-mutant cells suppress Class I MHC expression via global loss

609 of antigen processing and presenting machinery. a, Rank plot of all protein abundance

610 changes in KRAS ${ }^{\mathrm{G} 12 \mathrm{~V}}$-mutant cells compared to vector control, generated by LC-MS/MS. HLA-

$611 \mathrm{~A},-\mathrm{B},-\mathrm{C}$, and $-\mathrm{F}$ proteins are labeled in blue. b, Heat map showing HLA protein abundance in

612 each global proteome replicate. Replicates were clustered by unsupervised hierarchical

613 clustering using all detected proteins. c, Protein abundance of B2M in LC-MS/MS data. d, Top 
614 differentially abundant proteins between RIT1 ${ }^{\mathrm{WT}}$ cells and all other cell lines. Proteins are

615 ranked by the signal-to-noise (S2N) statistic, shown in the bar chart at the right. e, StringDB ${ }^{62}$

616 network analysis of proteins with $\mathrm{S} 2 \mathrm{~N}>2.5$ in analysis shown in $\mathbf{d}$. The network was significantly

617 more connected than expected by chance $(p<1 \mathrm{e}-16)$. Disconnected nodes, single connected

618 nodes, and disconnected clusters have been removed from the visualization. Edges represent

619 high confidence interaction scores (>0.9) and network edge thickness indicates the strength of

620 data support from all StringDB active interaction sources. f, Protein abundance of PSMB9 in LC-

621 MS/MS data. g, Global proteome-transcriptome correlation analysis. A dashed diagonal line

622 displays the linear regression generated by comparing the LFC of each gene in the

623 transcriptome to its respective protein LFC in the proteome. The resulting Pearson correlation

624 coefficient $(r)$ is shown. Genes outside the 95\% prediction interval are plotted in red, and include

625 HLA genes, metallothioneins, and intermediate filament proteins Vimentin (VIM) and alpha

626 internexin (INA).

627 Figure 5. Phosphoproteomic profiling illuminates novel differential post-translational

628 modifications in RIT1 ${ }^{\text {M90I- }}$ and KRAS-mutant cells. a, KSEA analysis of AALE

629 phosphoproteomes. Top differentially phosphorylated kinase substrates are shown. The full

630 KSEA results are shown in Supplementary Fig 4b-c. b, Violin plot of phospho-site abundance

631 of phospho-sites that are RPS6KA1 substrates. c, Violin plot of phospho-site abundance of

632 phospho-sites that are MAPKAPK2 substrates. d, Marker selection analysis identifies

633 differentially phosphorylated sites in KRAS-mutant cells compared to RIT1-mutant cells.

634 Phosphosites from KRAS-mutant and RIT1 ${ }^{\text {M90I }}$-mutant replicate-level phosphoproteome profiles

635 (Supplementary File 2) were compared by two-tailed t-test. The top 20 significantly (FDR <

636 0.05) differentially phosphorylated sites in each direction are shown and ranked by t-statistic. A

637 heat map displays the LFC in phosphorylated peptide abundance of each site compared to

638 vector control, after normalizing to total protein abundance. e, LFC of EGFR phosphosites in 
639 KRAS-mutant and RIT1-mutant cells. Data shown is the mean + SD of $n=8$ KRAS-mutant

640 replicates and $n=4$ RIT1-mutant replicates. *, FDR $<0.01$ as determined by two-tailed t-test and

641 two-stage linear step-up procedure of Benjamini, Krieger and Yekutieli. f, Relative

642 phosphorylation of USO1 at serine 48 as determined by LC-MS/MS. Box and whiskers show the

643 25th-75th percentiles and minimum to maximum of the data, respectively. $\mathbf{g}$, Relationship of

644 USO1 S48 phosphorylation to KRAS total protein abundance. A dashed line displays the linear

645 regression fit and gray lines display the 95\% confidence interval of the linear model. $r=0.70, p$

$646<0.01$

SUPPLEMENTARY FIGURE LEGENDS

Supplementary Figure 1. Workflow and quality control of proteomic and transcriptomic

profiling. a, Replicate-level workflow for tandem mass tag (TMT) labeling and LC-MS/MS.

with control samples used to link the two sets. b, TMT 10-plex layout showing mass tags associated with each replicate. c, Average pairwise replicate correlations (Pearson $r$ ) of all

654 replicates from each sample group indicated. d, Enrichment analysis of differentially expressed

655 genes between KRAS or RIT1 perturbed lines and vector controls using goseq ${ }^{56}$. mSigDB

656 hallmark gene sets specific to KRAS signaling are shown. e, RNA-seq run and mapping

657 statistics show total reads, mapped reads, and reads mapped to rRNA, for each sample.

659 Supplementary Figure 2. RIT1 and KRAS promote epithelial-to-mesenchymal transition.

660 a, Changes in mRNA transcript levels of EMT genes VIM, CDH2, FN1, and KRT19, in each

661 isogenic cell line compared to vector control. LFC, $\log _{2}$ (fold-change) compared to vector cells. b,

662 Transcription factor target enrichment analysis using Enrichr libraries of differentially expressed

663 genes in RIT1 ${ }^{\mathrm{WT}}, \mathrm{KRAS}^{\mathrm{WT}}$, and $\mathrm{KRAS}^{\mathrm{Q61H}}$-mutant cells. FET, Fisher's exact test. Red = Snail 
664 family. Orange $=$ confirmed EMT genes in $\mathrm{dbEMT}^{59}$. Pale orange $=\mathrm{EMT}$-associated genes in 665 literature.

667 Supplementary Figure 3. Post-transcriptional loss of Class I MHC proteins. a, Rank plot of 668 all protein abundance changes in $\mathrm{KRAS}^{\mathrm{Q} 61 \mathrm{H}}$-mutant or RIT1 ${ }^{\mathrm{M} 90 \mathrm{I}}$-mutant cells compared to vector 669 control, generated by LC-MS/MS. HLA-A,-B,-C, and -F proteins are labeled. LFC, $\log _{2}$ fold670 change. $\mathbf{b}$, Change in mRNA transcript levels of HLA genes and upstream regulators of MHC

671 Class I, in each isogenic cell line compared to vector controls. LFC, $\log _{2}$ fold change compared 672 to vector cells. c, Correlation of protein levels in HLA-A and PSMB9 across each isogenic cell 673 line. A line is the best-fit linear regression with significant non-zero slope $(p<0.05)$.

677 replicate correlation (Pearson $r$ ) heatmap and unsupervised clustering of phosphoproteome 678 data. b, KSEA of phosphoproteome data for RIT1 $1^{\mathrm{WT}}$ and RIT1 ${ }^{\mathrm{M} 90 \mathrm{l}}$-expressing cells. The kinase 679 z-score indicates the overall score for each kinase listed, normalized by the total number of 680 substrates. Significant scores $(p<0.05)$ are indicated in red and blue. Phospho-sites of kinases 681 in red were more highly abundant in the cell line compared to vector control, whereas phospho682 sites of kinases in blue were more highly abundant in vector control than the indicated cell line. 683 c, KSEA of phosphoproteome data for KRAS-expressing cells. Labeling as in (b). d, Violin plot 684 of phospho-site abundance of AKT1 substrate sites. e, Violin plot of phospho-site abundance of 685 PRKCA substrate sites. f, Violin plot of phospho-site abundance of AURKB substrate sites. $\mathbf{g}$, 686 EGFR protein abundance in LC-MS/MS data compared to vector control. f, Proportion of 687 phosphorylated sites in AHNAK proteins with differential phosphorylation between KRAS-mutant 688 and RIT1 ${ }^{\mathrm{M} 90 \mathrm{I}}$-mutant cells. Data shown is the percentage of differentially abundant 
bioRxiv preprint doi: https://doi.org/10.1101/2020.04.10.030460; this version posted April 11, 2020. The copyright holder for this preprint (which was not certified by peer review) is the author/funder, who has granted bioRxiv a license to display the preprint in perpetuity. It is made available under aCC-BY-NC-ND 4.0 International license.

689 phosphorylation sites in AHNAK and AHNAK2 compared to all other sites. Significance was

690 determined from the analysis in (b), FDR $<0.05 .{ }^{* * *}, p<0.0001$ by two-sided Fisher's exact

691 test.

692 


\section{References}

694

1. Trahey, M. \& McCormick, F. A cytoplasmic protein stimulates normal N-ras p21 GTPase, but does not affect oncogenic mutants. Science 238, 542-545 (1987).

2. Emil F. Pai, Wolfgang Kabsch, Ute Krengel, Kenneth C. Holmes, Jacob John \& Alfred Wittinghofer. Structure of the guanine-nucleotide-binding domain of the Ha-ras oncogene p21 in the triphosphate conformation. Nature 2, 1105-1111 (1989).

3. Nakano, H. et al. Isolation of transforming sequences of two human lung carcinomas: structural and functional analysis of the activated c-K-ras oncogenes. Proc. Natl. Acad. Sci. U. S. A. 81, 71-75 (1984).

4. Rodenhuis, S. et al. Mutational activation of the K-ras oncogene. A possible pathogenetic factor in adenocarcinoma of the lung. N. Engl. J. Med. 317, 929-935 (1987).

5. Berger, A. H. et al. Oncogenic RIT1 mutations in lung adenocarcinoma. Oncogene 33, 4418-4423 (2014).

6. Gómez-Seguí, I. et al. Novel recurrent mutations in the RAS-like GTP-binding gene RIT1 in myeloid malignancies. Leukemia 27, 1943-1946 (2013).

7. Aoki, Y. et al. Gain-of-Function Mutations in RIT1 Cause Noonan Syndrome, a RAS/MAPK Pathway Syndrome. Am. J. Hum. Genet. 93, 173-180 (2013).

8. Cai, W. et al. Rit GTPase signaling promotes immature hippocampal neuronal survival. J. Neurosci. 32, 9887-9897 (2012).

9. Rusyn, E. V. et al. Rit, a non-lipid-modi/Eed Ras-related protein, transforms NIH3T3 cells without activating the ERK, JNK, p38 MAPK or PI3K/Akt pathways. Oncogene 19, 46854694 (2000).

10. Sakabe, K. et al. Potent transforming activity of the small GTP-binding protein Rit in NIH 3T3 cells: evidence for a role of a p38gamma-dependent signaling pathway. FEBS Lett. 511, 15-20 (2002). 
719 11. Castel, P. et al. RIT1 oncoproteins escape LZTR1-mediated proteolysis. Science 363,

$720 \quad 1226-1230(2019)$.

721 12. Fang, Z. et al. Biochemical Classification of Disease-associated Mutants of RAS-like

722 Protein Expressed in Many Tissues (RIT1). J. Biol. Chem. 291, 15641-15652 (2016).

723 13. Lundberg, A. S. et al. Immortalization and transformation of primary human airway epithelial

724 cells by gene transfer. Oncogene 21, 4577-4586 (2002).

725 14. Simanshu, D. K., Nissley, D. V. \& McCormick, F. RAS Proteins and Their Regulators in

$726 \quad$ Human Disease. Cell 170, 17-33 (2017).

727 15. Young, M. R. \& Colburn, N. H. Fra-1 a target for cancer prevention or intervention. Gene

$728 \quad 379,1-11(2006)$.

729 16. Mechta, F., Lallemand, D., Pfarr, C. M. \& Yaniv, M. Transformation by ras modifies AP1

730 composition and activity. Oncogene 14, 837-847 (1997).

731 17. Casalino, L., De Cesare, D. \& Verde, P. Accumulation of Fra-1 in ras-transformed cells

732 depends on both transcriptional autoregulation and MEK-dependent posttranslational

733 stabilization. Mol. Cell. Biol. 23, 4401-4415 (2003).

734 18. Yoshihara, E. et al. Thioredoxin/Txnip: redoxisome, as a redox switch for the pathogenesis

735 of diseases. Front. Immunol. 4, 514 (2014).

736 19. Parikh, H. et al. TXNIP regulates peripheral glucose metabolism in humans. PLoS Med. 4, $737 \quad$ e158 (2007).

738 20. Elgort, M. G., O’Shea, J. M., Jiang, Y. \& Ayer, D. E. Transcriptional and Translational

739 Downregulation of Thioredoxin Interacting Protein Is Required for Metabolic

$740 \quad$ Reprogramming during G(1). Genes Cancer 1, 893-907 (2010).

741 21. Ye, Z. \& Ayer, D. E. Ras Suppresses TXNIP Expression by Restricting Ribosome

742 Translocation. Mol. Cell. Biol. 38, (2018).

743 22. Liberzon, A. et al. The Molecular Signatures Database (MSigDB) hallmark gene set 
collection. Cell Syst 1, 417-425 (2015).

23. Ye, X. \& Weinberg, R. A. Epithelial-Mesenchymal Plasticity: A Central Regulator of Cancer Progression. Trends Cell Biol. 25, 675-686 (2015).

24. Singh, A. et al. A gene expression signature associated with 'K-Ras addiction' reveals regulators of EMT and tumor cell survival. Cancer Cell 15, 489-500 (2009).

25. Shao, D. D. et al. KRAS and YAP1 converge to regulate EMT and tumor survival. Cell 158, 171-184 (2014).

26. Shukla, S. A. et al. Comprehensive analysis of cancer-associated somatic mutations in class I HLA genes. Nat. Biotechnol. 33, 1152-1158 (2015). response to immune checkpoint therapies for cancer: implications for precision medicine. Genome Med. 10, 93 (2018).

28. Gettinger, S. et al. Impaired HLA Class I Antigen Processing and Presentation as a Mechanism of Acquired Resistance to Immune Checkpoint Inhibitors in Lung Cancer. Cancer Discov. 7, 1420-1435 (2017).

29. Jongsma, M. L. M., Guarda, G. \& Spaapen, R. M. The regulatory network behind MHC class I expression. Mol. Immunol. 113, 16-21 (2019).

30. Burr, M. L. et al. An Evolutionarily Conserved Function of Polycomb Silences the MHC Class I Antigen Presentation Pathway and Enables Immune Evasion in Cancer. Cancer

31. Seliger, B. et al. Down-regulation of the MHC class I antigen-processing machinery after oncogenic transformation of murine fibroblasts. Eur. J. Immunol. 28, 122-133 (1998). phosphorylation via RSK and stimulates cap-dependent translation. J. Biol. Chem. 282, 14056-14064 (2007). 
33. Mendoza, M. C., Er, E. E. \& Blenis, J. The Ras-ERK and PI3K-mTOR pathways: cross-talk and compensation. Trends Biochem. Sci. 36, 320-328 (2011).

34. Wiredja, D. D., Koyutürk, M. \& Chance, M. R. The KSEA App: a web-based tool for kinase activity inference from quantitative phosphoproteomics. Bioinformatics (2017) doi:10.1093/bioinformatics/btx415.

35. Hornbeck, P. V. et al. PhosphoSitePlus: a comprehensive resource for investigating the structure and function of experimentally determined post-translational modifications in man and mouse. Nucleic Acids Res. 40, D261-D270 (2011).

36. Linding, R. et al. Systematic discovery of in vivo phosphorylation networks. Cell 129, 14151426 (2007).

37. Unni, A. M., Lockwood, W. W., Zejnullahu, K., Lee-Lin, S.-Q. \& Varmus, H. Evidence that synthetic lethality underlies the mutual exclusivity of oncogenic KRAS and EGFR mutations in lung adenocarcinoma. Elife 4, e06907 (2015).

38. Chang, C. P. et al. Ligand-induced internalization of the epidermal growth factor receptor is mediated by multiple endocytic codes analogous to the tyrosine motif found in constitutively internalized receptors. J. Biol. Chem. 268, 19312-19320 (1993).

39. Tong, J., Taylor, P., Peterman, S. M., Prakash, A. \& Moran, M. F. Epidermal growth factor receptor phosphorylation sites Ser991 and Tyr998 are implicated in the regulation of receptor endocytosis and phosphorylations at Ser1039 and Thr1041. Mol. Cell. Proteomics

41. Prior, I. A. \& Hancock, J. F. Ras trafficking, localization and compartmentalized signalling. Semin. Cell Dev. Biol. 23, 145-153 (2012).

793 42. Shao, H., Kadono-Okuda, K., Finlin, B. S. \& Andres, D. A. Biochemical characterization of 
the Ras-related GTPases Rit and Rin. Arch. Biochem. Biophys. 371, 207-219 (1999).

43. Park, J. W. et al. AHNAK Loss in Mice Promotes Type II Pneumocyte Hyperplasia and Lung Tumor Development. Mol. Cancer Res. 16, 1287-1298 (2018).

44. Lee, I. H. et al. Ahnak functions as a tumor suppressor via modulation of TGF $\beta / S m a d$ signaling pathway. Oncogene 33, 4675-4684 (2014).

45. Che, Y. et al. KRAS regulation by small non-coding RNAs and SNARE proteins. Nat. Commun. 10, 5118 (2019).

46. Ritchie, C. et al. Analysis of K-Ras Interactions by Biotin Ligase Tagging. Cancer Genomics Proteomics 14, 225-239 (2017).

47. Ambrogio, C. et al. KRAS Dimerization Impacts MEK Inhibitor Sensitivity and Oncogenic Activity of Mutant KRAS. Cell 172, 857-868.e15 (2018).

48. Pylayeva-Gupta, Y., Grabocka, E. \& Bar-Sagi, D. RAS oncogenes: weaving a tumorigenic web. Nat. Rev. Cancer 11, 761-774 (2011).

49. Carpenter, A. E. et al. CellProfiler: image analysis software for identifying and quantifying cell phenotypes. Genome Biol. 7, R100 (2006).

50. Dobin, A. et al. STAR: ultrafast universal RNA-seq aligner. Bioinformatics 29, 15-21 (2013).

51. Picard Tools - By Broad Institute. http://broadinstitute.github.io/picard.

52. Wang, L., Wang, S. \& Li, W. RSeQC: quality control of RNA-seq experiments. Bioinformatics 28, 2184-2185 (2012).

813 53. Liao, Y., Smyth, G. K. \& Shi, W. FeatureCounts: An efficient general purpose program for 814 assigning sequence reads to genomic features. Bioinformatics 30, 923-930 (2014).

815 54. Robinson, M. D., McCarthy, D. J. \& Smyth, G. K. edgeR: A Bioconductor package for 816 differential expression analysis of digital gene expression data. Bioinformatics 26, 139-140 817 (2009).

818 55. Mertins, P. et al. Reproducible workflow for multiplexed deep-scale proteome and 
phosphoproteome analysis of tumor tissues by liquid chromatography-mass spectrometry. Nat. Protoc. 13, 1632-1661 (2018).

56. Young, M. D., Wakefield, M. J., Smyth, G. K. \& Oshlack, A. Gene ontology analysis for RNA-seq: accounting for selection bias. Genome Biol. 11, R14 (2010).

57. Subramanian, A. et al. Gene set enrichment analysis: a knowledge-based approach for interpreting genome-wide expression profiles. Proc. Natl. Acad. Sci. U. S. A. 102, 1554515550 (2005).

58. Keenan, A. B. et al. ChEA3: transcription factor enrichment analysis by orthogonal omics integration. Nucleic Acids Res. 47, W212-W224 (2019).

59. Zhao, M., Liu, Y., Zheng, C. \& Qu, H. dbEMT 2.0: An updated database for epithelialmesenchymal transition genes with experimentally verified information and precalculated regulation information for cancer metastasis. J. Genet. Genomics 46, 595-597 (2019).

60. Casado, P. et al. Kinase-substrate enrichment analysis provides insights into the heterogeneity of signaling pathway activation in leukemia cells. Sci. Signal. 6, rs6 (2013).

61. Larkin, M. A. et al. Clustal W and Clustal X version 2.0. Bioinformatics 23, 2947-2948 (2007).

62. Szklarczyk, D. et al. STRING v10: protein-protein interaction networks, integrated over the tree of life. Nucleic Acids Res. 43, D447-52 (2015). 

bioRxiv preprint doi: https://doi.org/10.1101/2020.04.10.030460; this version posted April 11, 2020. The copyright holder for this preprint (whiqfigure 1
was not certified by peer review) is the author/funder, who has granted bioRxiv a license to display the preprint in perpetuity. It is made available under aCC-BY-NC-ND 4.0 International license.

$\begin{array}{ll}: * * * * *: * * . *:: * * * * * * * . * *: & * * * * * \\ \text { Switch I domain } & \text { GTP binding } \star \text { RIT1 variant } \\ \text { Switch II domain } & \text { CAAX motif } \star \text { KRAS variant }\end{array}$

b

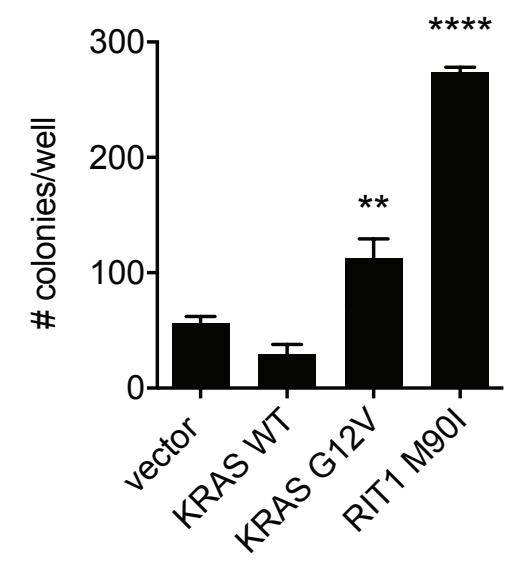

d

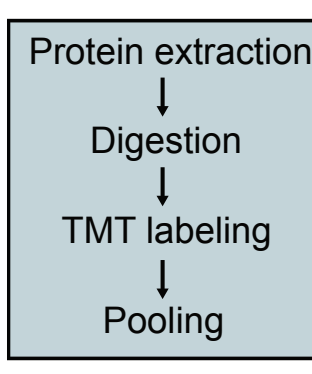

$(5 \%)$ global proteome

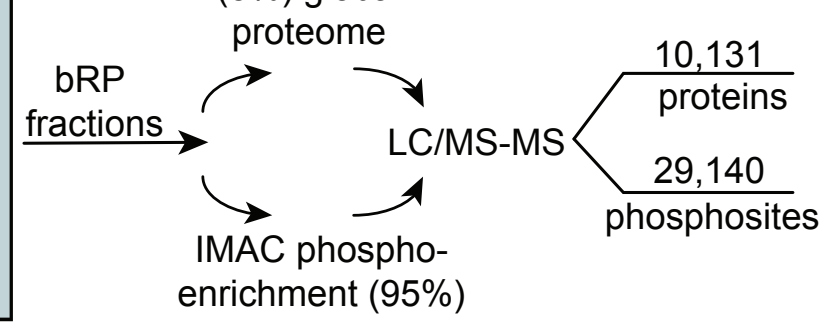

RIT1 RIT1 KRAS KRAS KRAS Vector WT M90I WT G12V Q61H

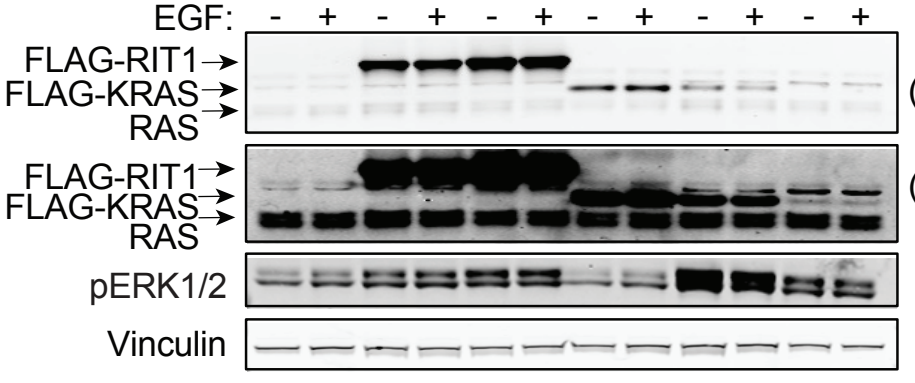

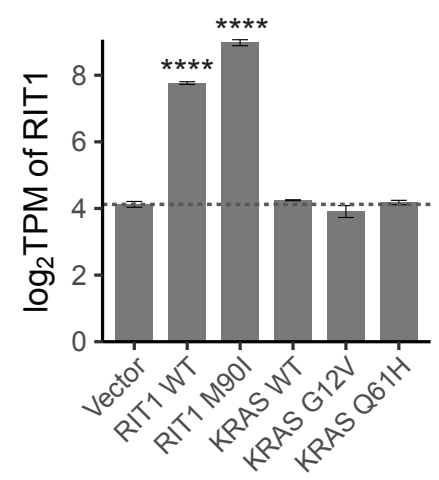
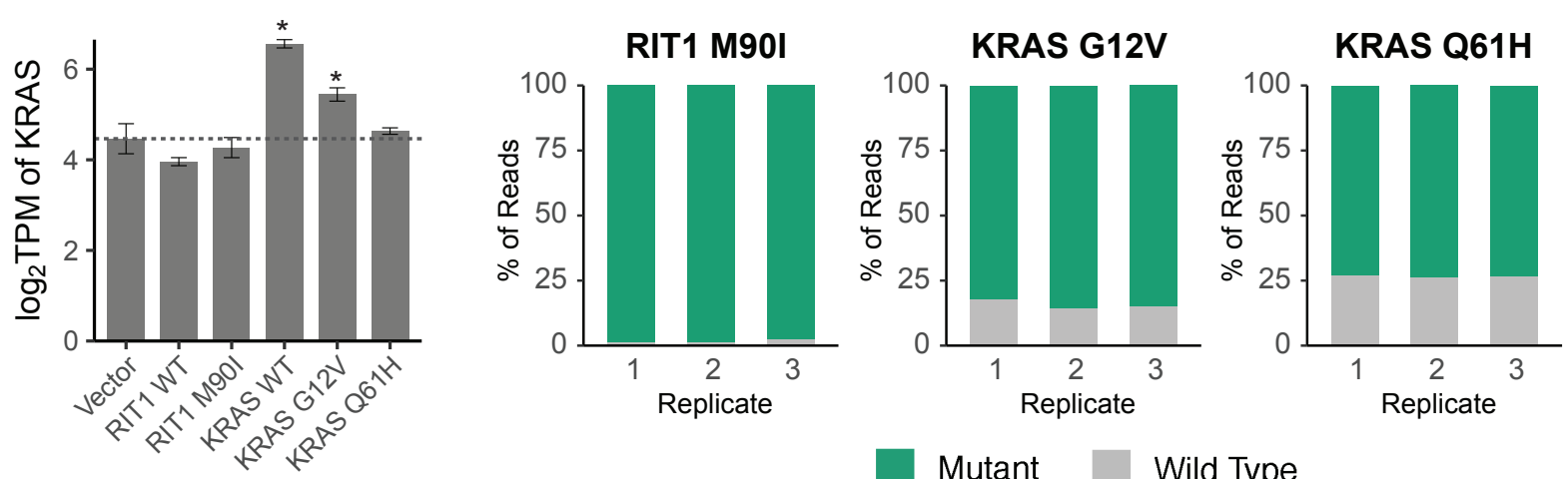

Mutant $\underset{\substack{70 \mathrm{M} \text { reads } \\ \text { per sample }}}{\stackrel{\text { RNA-seq }}{\longrightarrow}}$ Genes

Illumina TruSeq poly-A selection library Pooling

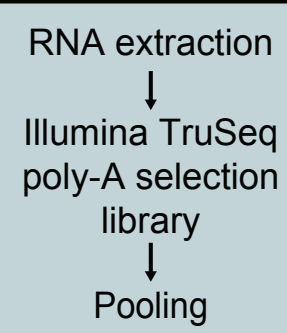


bioRxiv preprint doi: https://doi.org/10.1101/2020.04.10.030460; this version posted April 11, 2020. The copyright holder for this preprint (whiquigure 2
was not certified by peer review) is the author/funder, who has granted bioRxiv a license to display the preprint in perpetuity. It is made

a
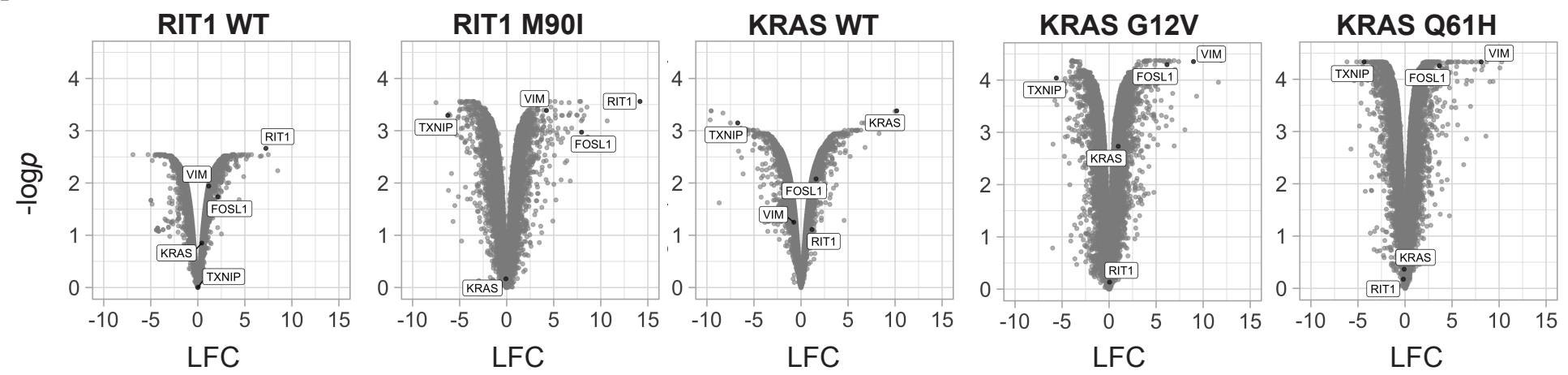

b

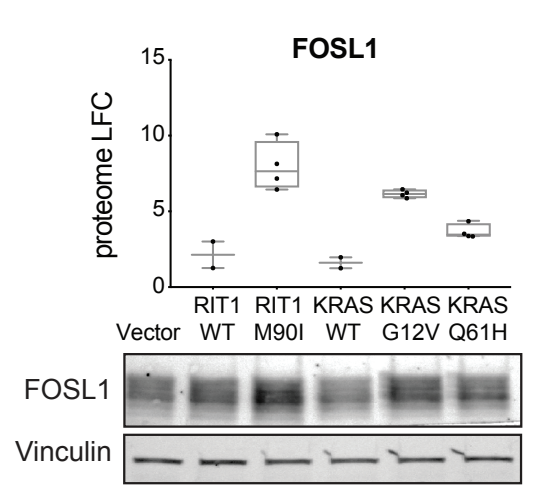

C

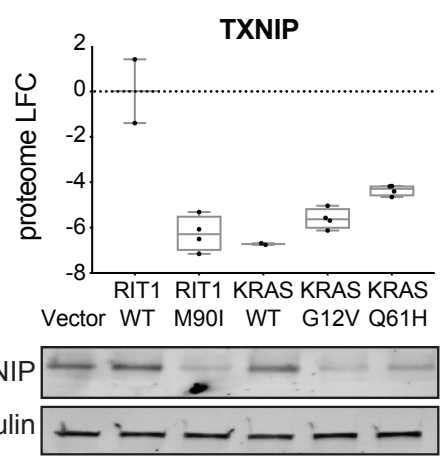

e

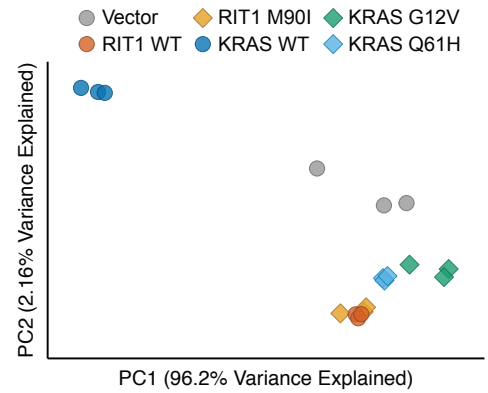

d

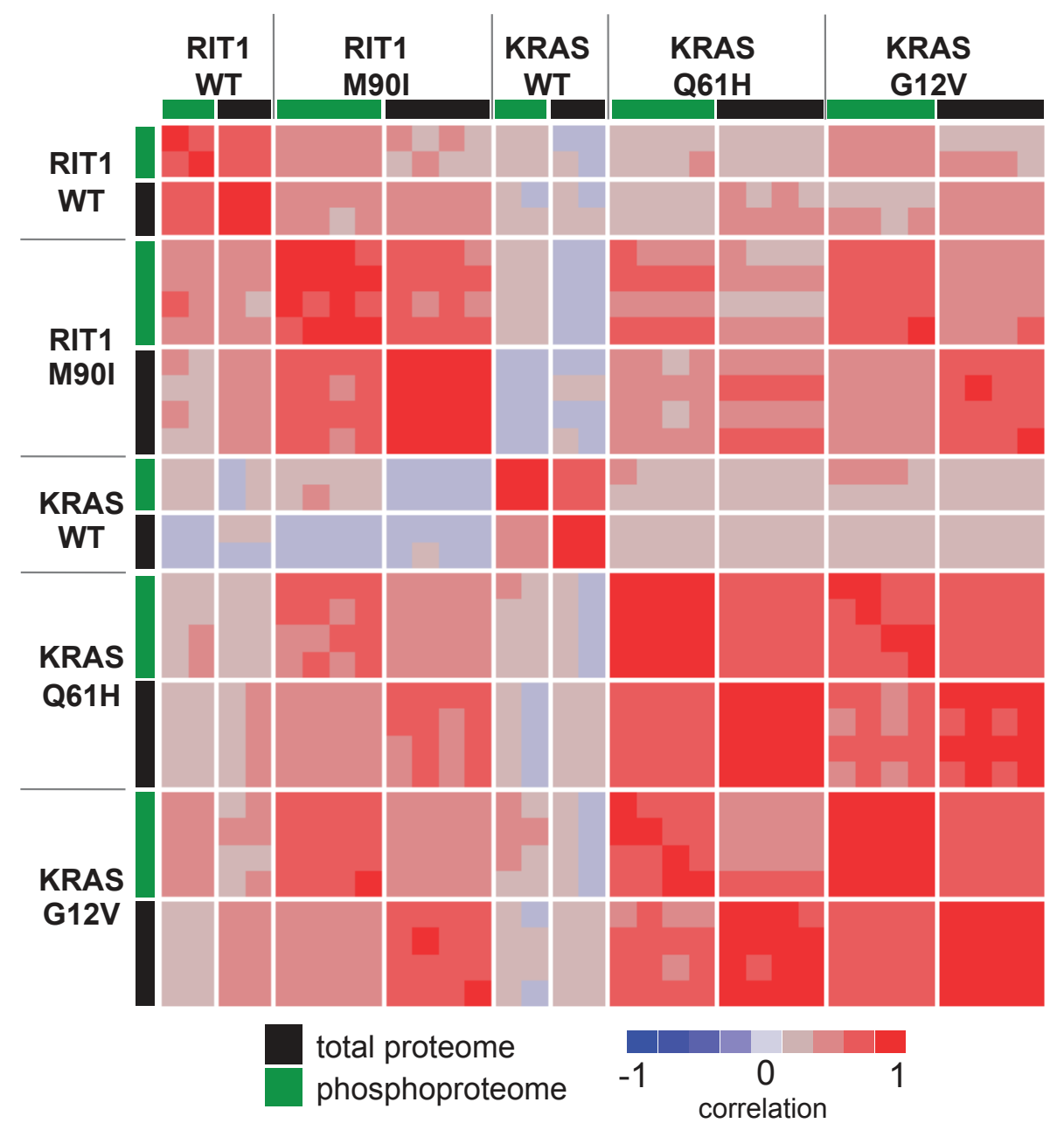



bioRxiv preprint doi: https://doi.org/10.1101/2020.04.10.030460; this version posted April 11, 2020. The copyright holder for this preprint (whiqh igure $\mathbf{3}$
was not certified by peer review) is the author/funder, who has granted bioRxiv a license to display the preprint in perpetuity. It is made
available under aCC-BY-NC-ND 4.0 International license.

a

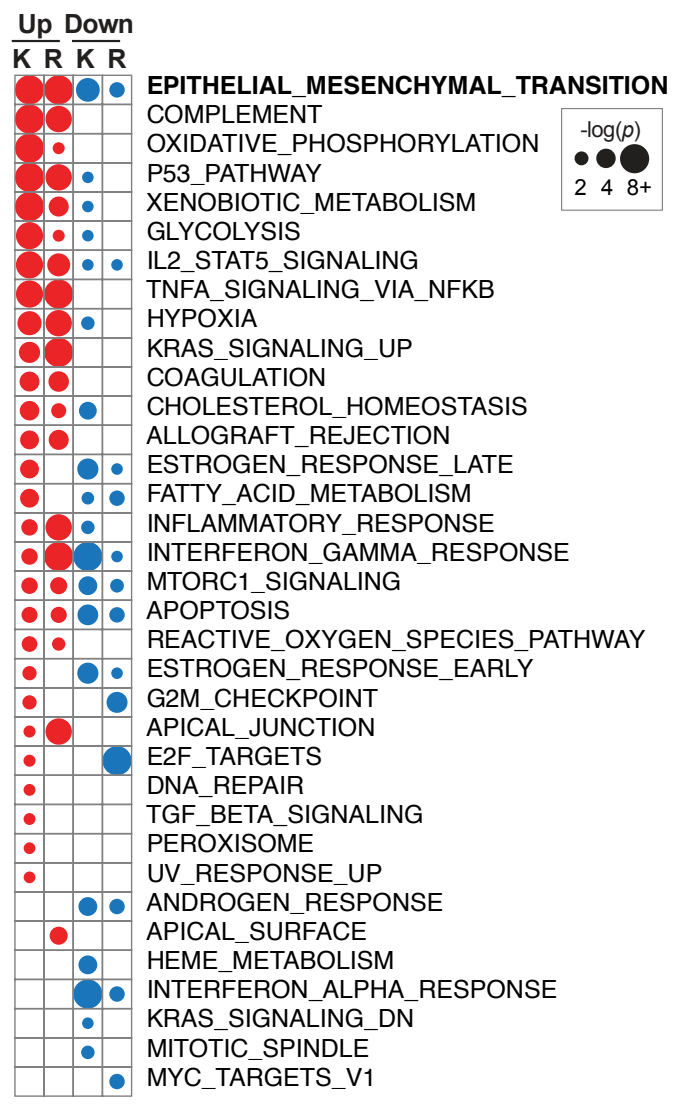

b
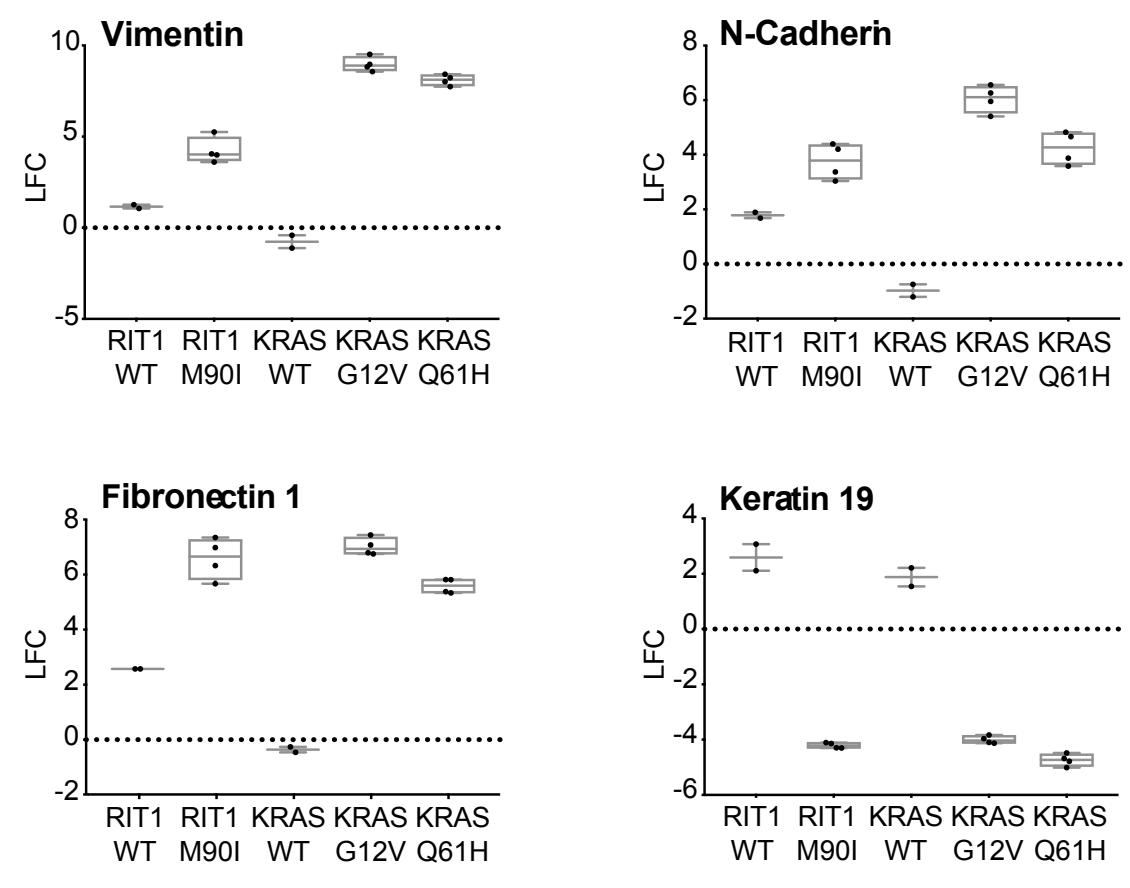
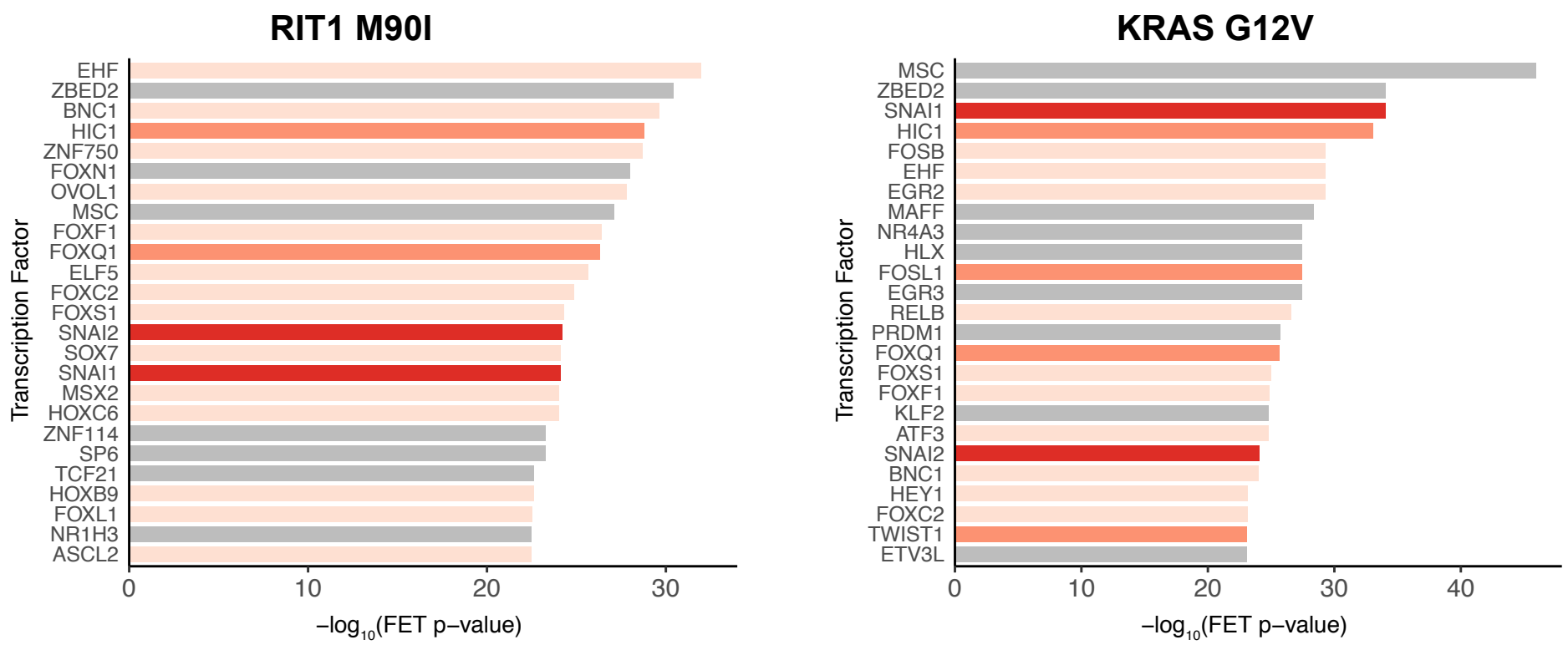

available under aCC-BY-NC-ND 4.0 International license.
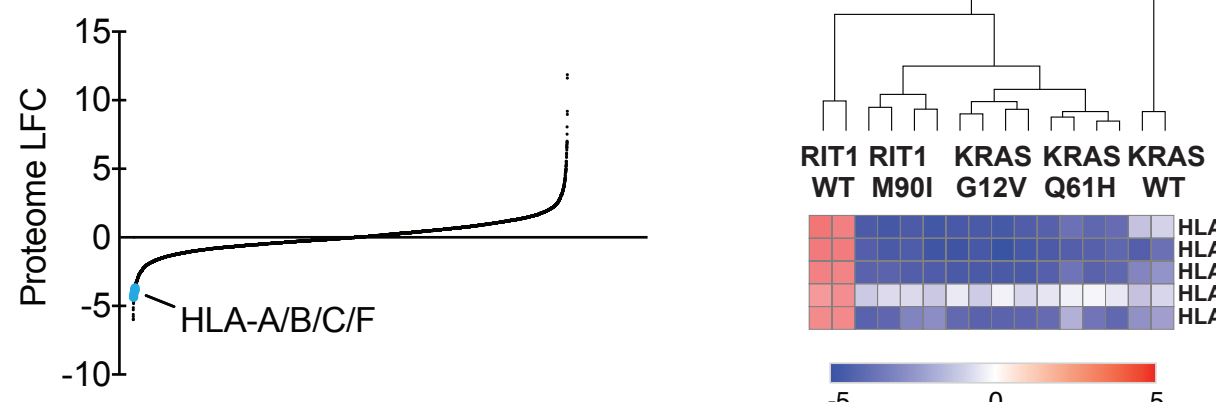

WT M90I G12V Q61H WT
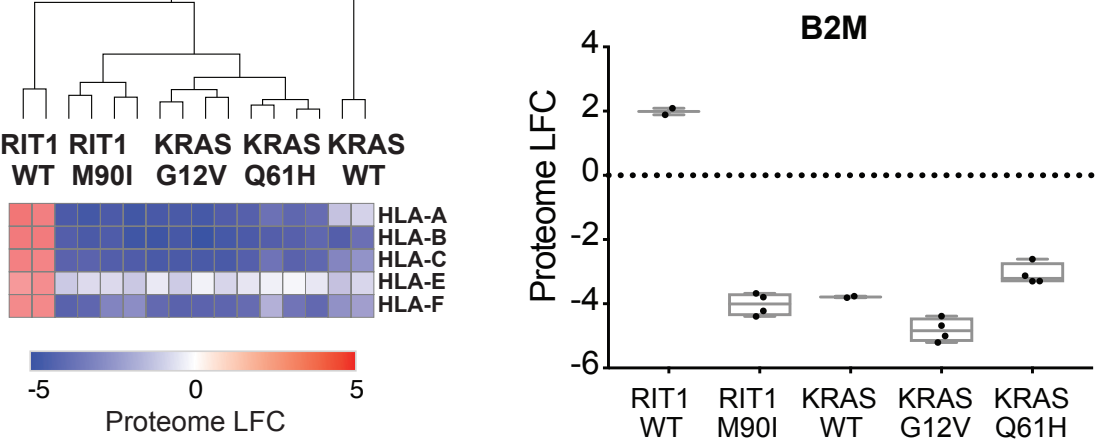

d

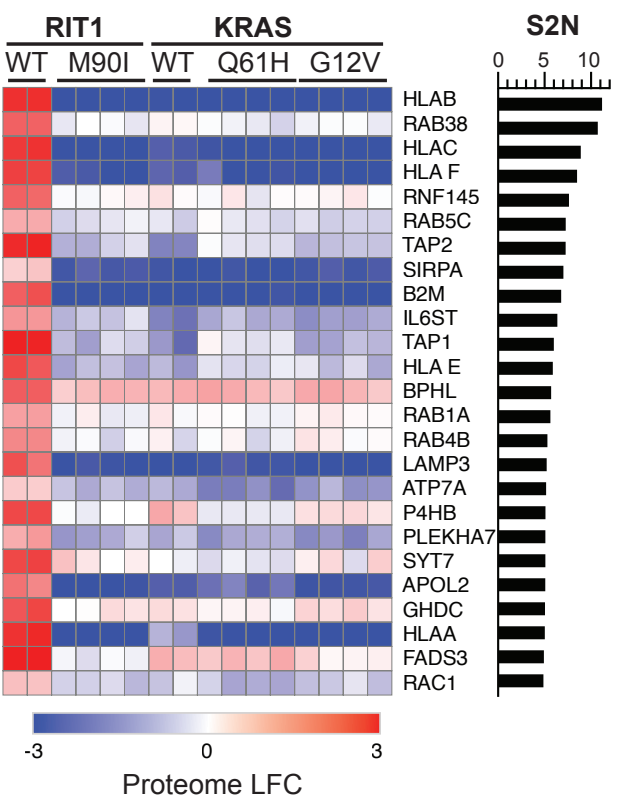

e

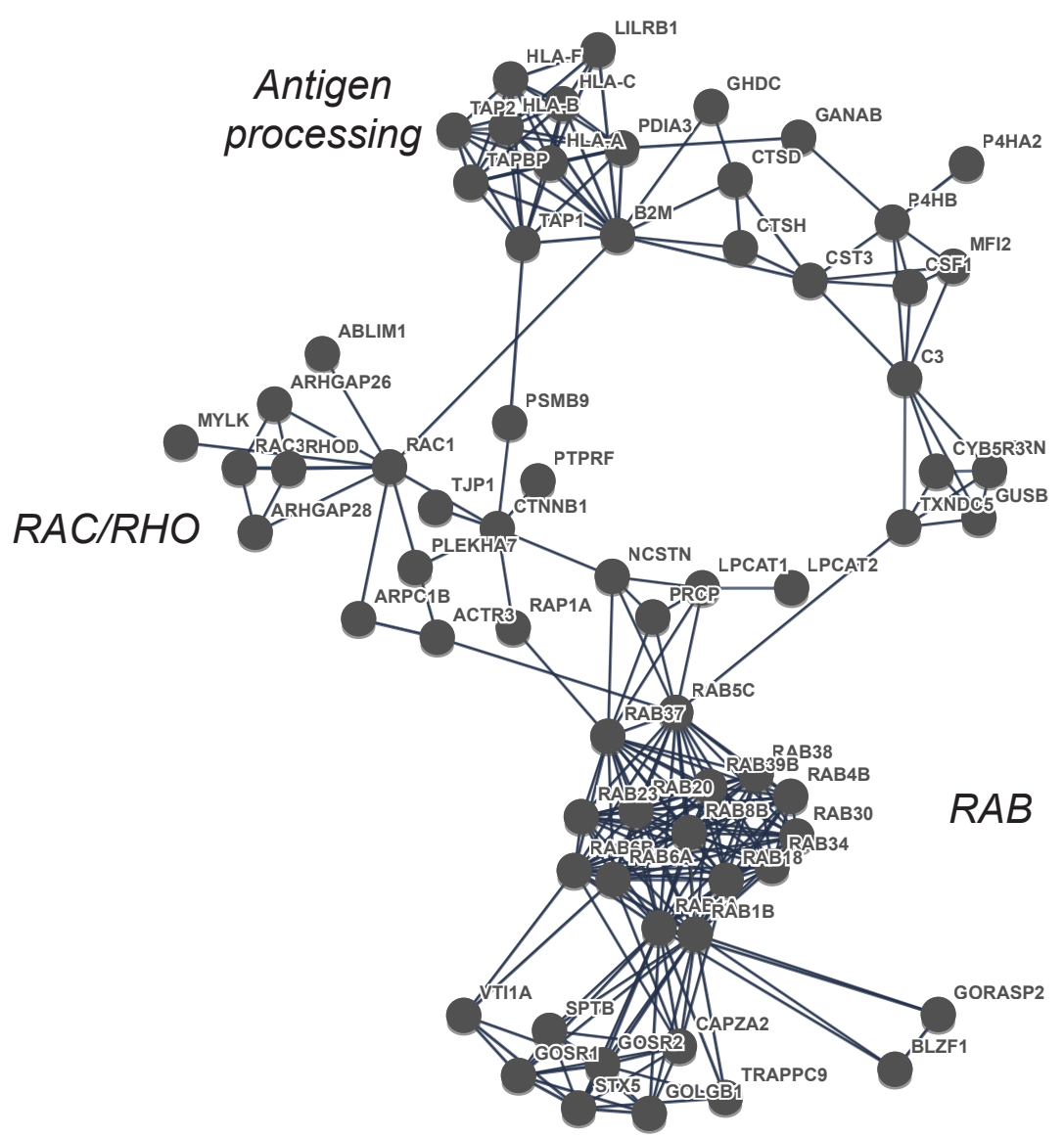

$\mathbf{f}$

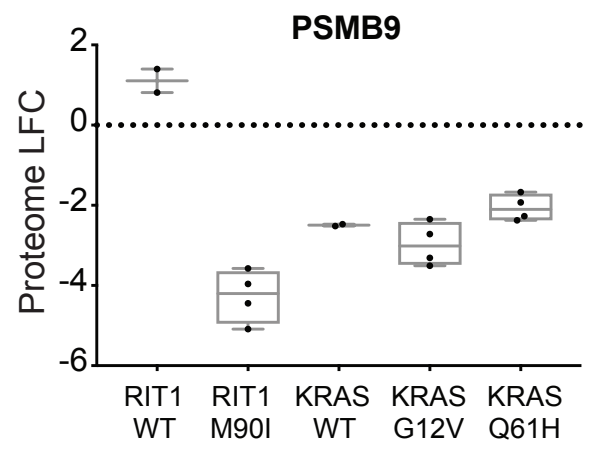

SNARE-binding

g

KRAS G12V

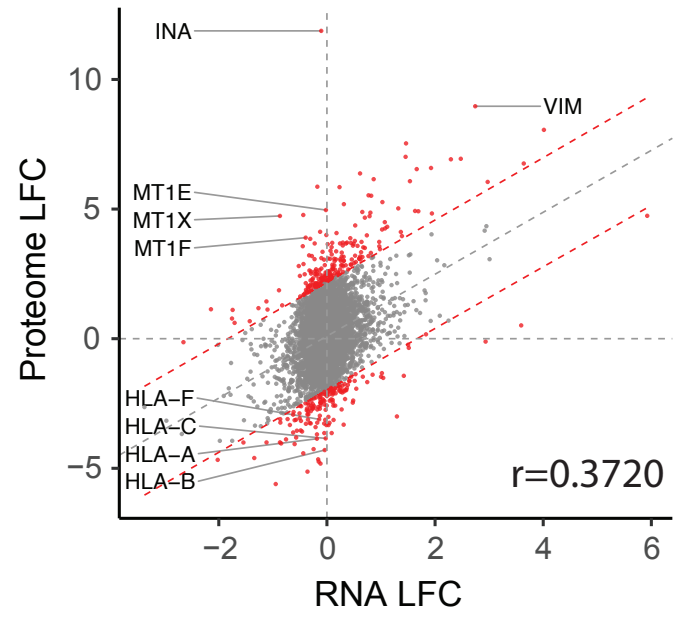

KRAS Q61H

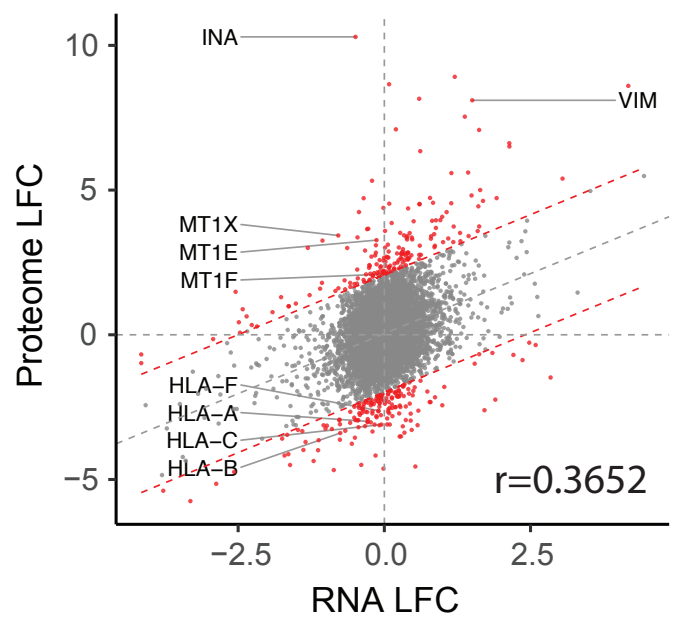


Figure 5

a

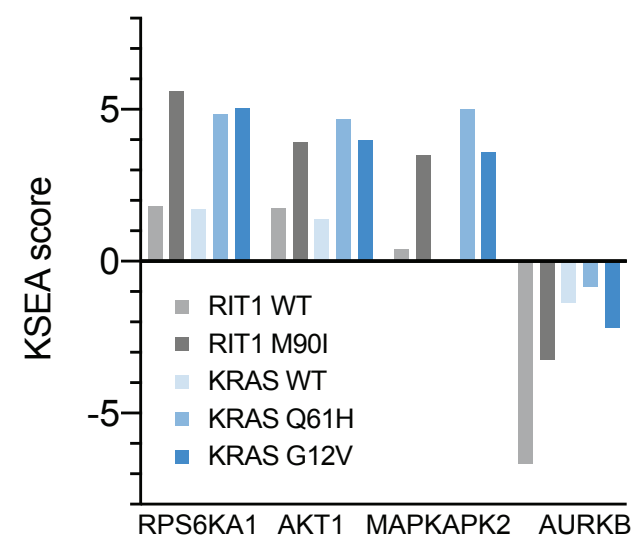

e

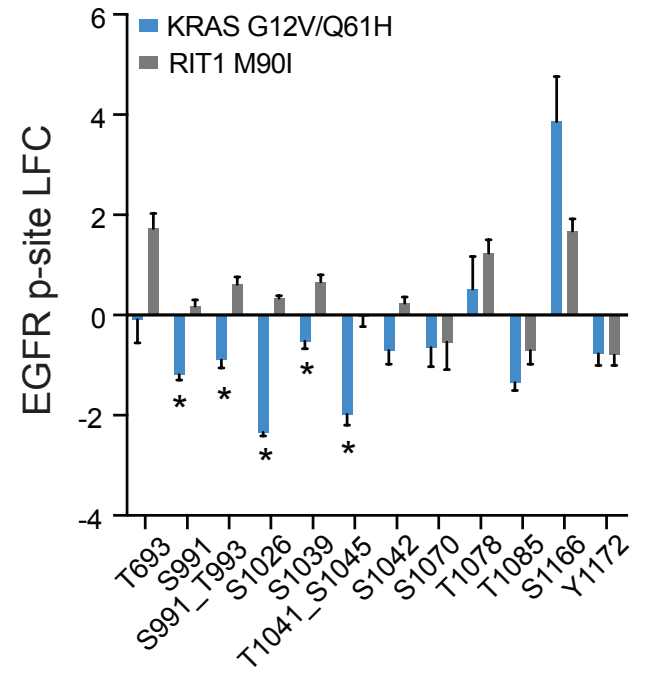

b

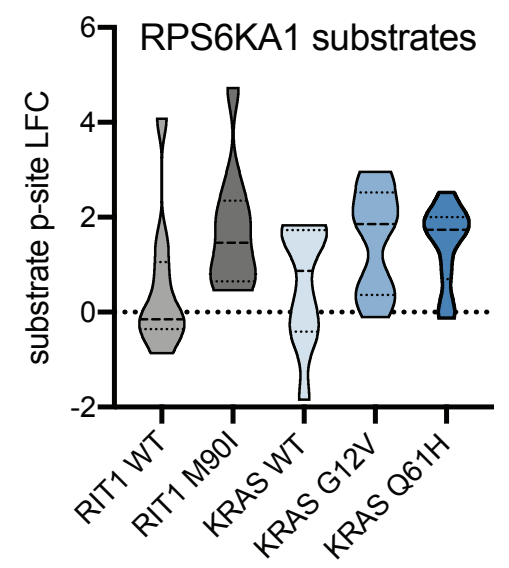

f

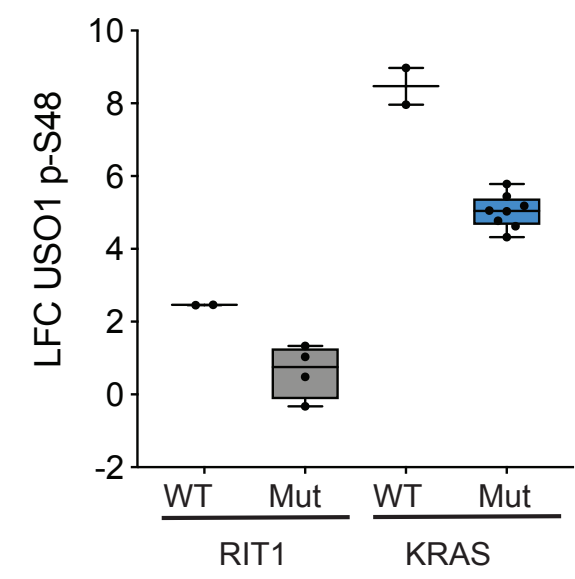

C

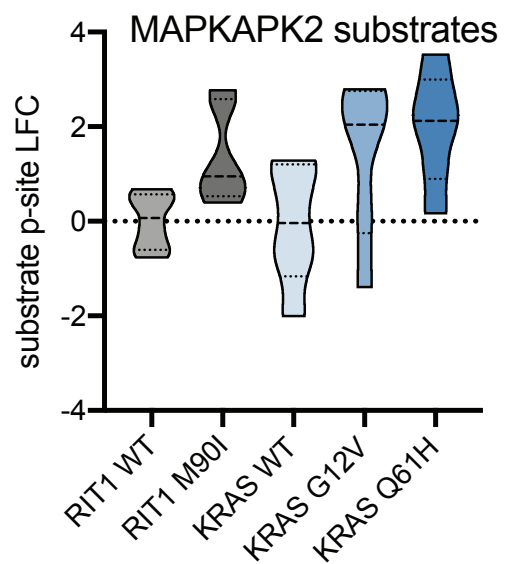

g

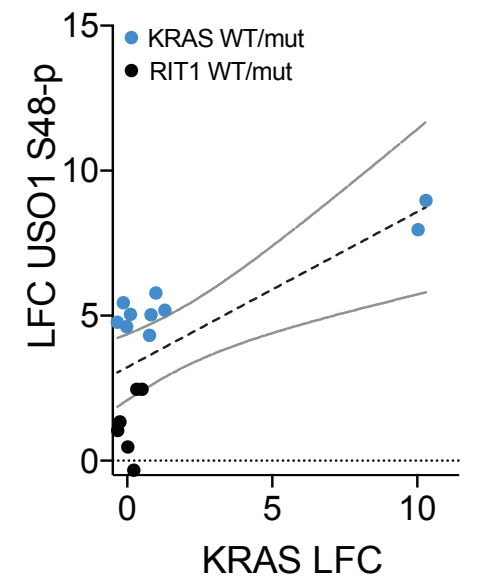

d

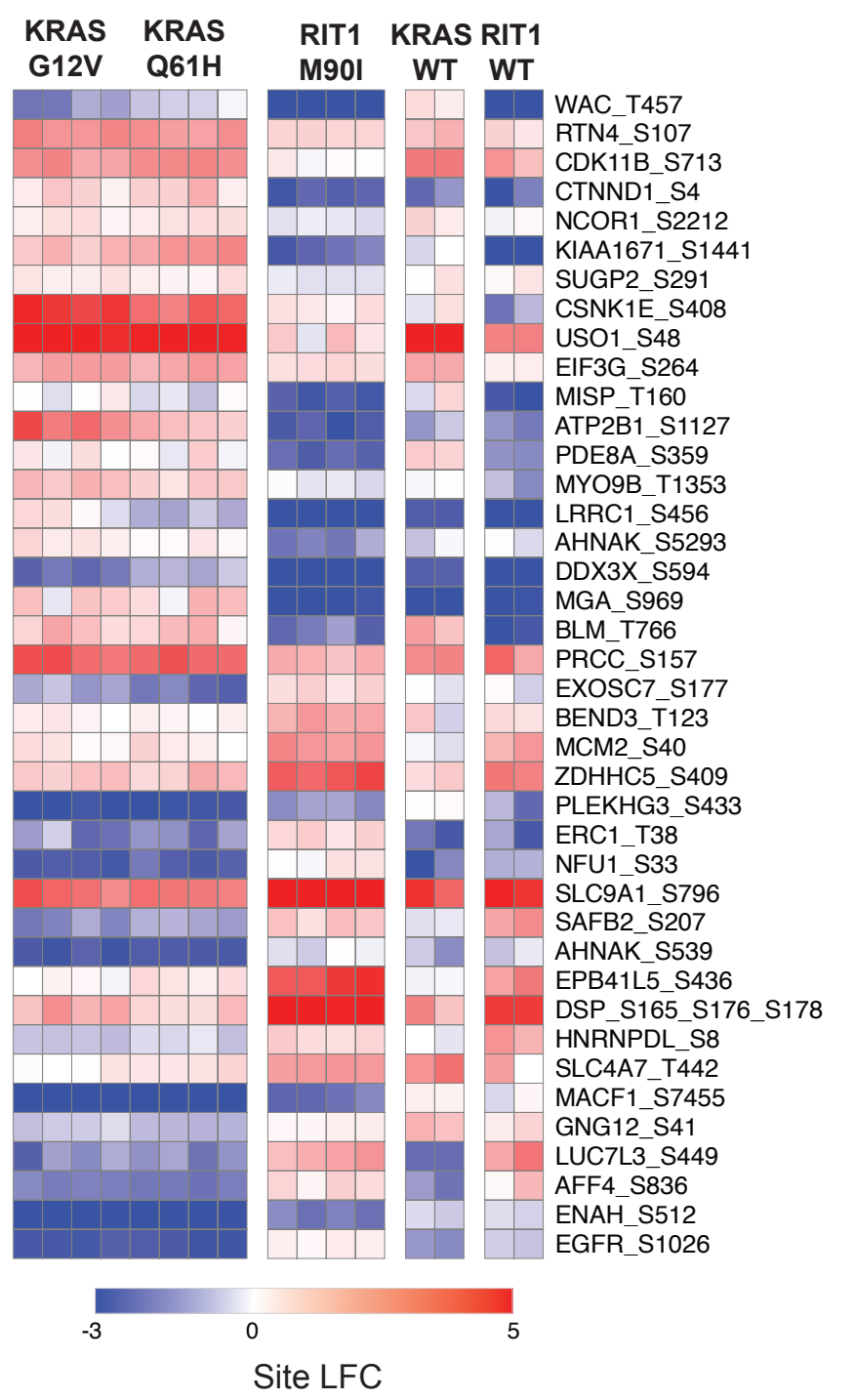

